\title{
Low-voltage and fully flexible analogue/digital mixed-signal circuits based on carbon nanotubes for epidermal sensor applications
}

\author{
Taikga Kashima \\ Nagoya University \\ Shigeru Kishimoto \\ Nagoya University \\ Jun Hirotani \\ Nagoya University \\ Hiromichi Kataura \\ National Institute of Advanced Industrial Science and Technology \\ Yutaka Ohno ( $\nabla$ yohno@nagoya-u.jp ) \\ Nagoya University
}

Article

Keywords:

Posted Date: September 11th, 2020

DOl: https://doi.org/10.21203/rs.3.rs-68702/v1

License: (c) (1) This work is licensed under a Creative Commons Attribution 4.0 International License. Read Full License 


\section{Low-voltage and fully flexible analogue/digital mixed-signal}

\section{2 circuits based on carbon nanotubes for epidermal sensor}

\section{3 applications}

4 Taiga Kashima $^{1}$, Shigeru Kishimoto ${ }^{1}$, Jun Hirotani ${ }^{1}$, Hiromichi Kataura ${ }^{2}$, and Yutaka Ohno ${ }^{1,3, *}$

$5{ }^{1}$ Department of Electronics, Nagoya University, Furo-cho, Chikusa-ku, Nagoya 464-8603, 6 Japan

$7 \quad{ }^{2}$ Nanomaterials Research Institute, National Institute of Advanced Industrial Science and 8 Technology, Tsukuba, Ibaraki, 305-8565, Japan

$9{ }^{3}$ Institute of Materials and Systems for Sustainability, Nagoya University, Furo-cho, Chikusa-

10 ku, Nagoya 464-8601, Japan

$11{ }^{*}$ Corresponding author e-mail: yohno@nagoya-u.jp

12 Epidermal electronics that can be mounted directly onto human skin to monitor biological 13 signals have wide applicability, such as in healthcare and sports. This requires integrating 14 various analogue and analogue/digital mixed-signal circuits on a flexible film as well as sensors. 15 Flexible analogue circuits based on oxide semiconductors or amorphous Si have been realised, but so far they do not allow robust digital signal transmission to an external system, and they

17 have a high operating voltage. Here, we report a low-voltage and fully flexible analogue/digital 18 mixed-signal circuits based on carbon nanotubes (CNTs). The key contribution is a novel 19 operational amplifier with a high gain and low operating voltage, which is important for 20 negative-feedback circuits and dramatically suppresses the influence of device variability and 21 instability. Fully flexible CNT-based mixed-signal circuits were realised monolithically on a 22 plastic film for the first time and demonstrated stable and continuous operation at a low supply 23 voltage. 
24 Because flexible wearable sensors can come in close contact with soft human tissue, they are suitable for long-term and non-invasive monitoring of biological signals in a wide range of fields such as medicine, healthcare, and sports (Figure 1a). Intensive research has resulted in the development of epidermal sensors to detect a wide range of biological signals, such as the electrocardiogram (ECG), ${ }^{1-4}$ electromyogram, ${ }^{5,6}$ sweating, ${ }^{7,8}$ body temperature, ${ }^{9,10}$ surface strain, ${ }^{11-13}$ and pressure. ${ }^{14,15}$ However, these sensor systems process signals by relying on either a remote information terminal or rigid silicon-based chips placed on the sensor device, which degrades the overall flexibility and durability (Figure 1b). Replacing silicon chips with fully flexible electronics can help realise epidermal devices that provide intimate contact with the 33 human body.

34 To realise an epidermal sensor system, various electronic circuits need to be integrated on a flexible film, including the analogue frontend (AFE) and radiofrequency (RF) frontend (Figure 1c). The AFE amplifies and digitises the weak output signal from a sensor. Digital data are transferred to the RF frontend for wireless signal transmission. Flexible AFE circuits based on $\mathrm{IGZO}^{16-18}$ or amorphous $\mathrm{Si}^{19}$ have previously been reported, but they were not equipped with an analogue/digital converter (ADC), which is indispensable for robust signal transmission with a high signal-to-noise ratio (SNR) while excluding external noise. In addition, such AFE circuits required high power supply voltages of 10-55 V, which can be attributed to the high operating voltage as well as the variability and instability of their thin-film transistors (TFTs). The power supply voltage for a circuit is generally increased to obtain an operating margin to overcome device variability and instability. However, a low operating voltage is essential for wearable electronics to accommodate safety concerns and a limited power supply. 
a b

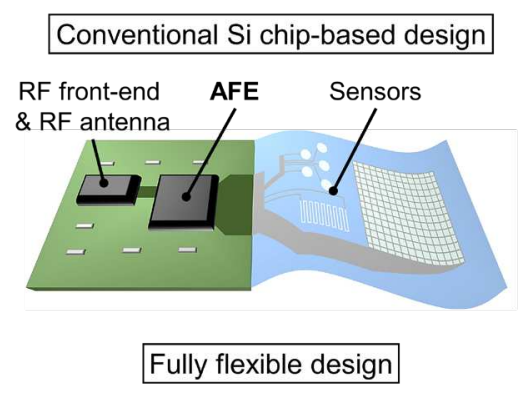

Figure 1 | Fully flexible electronics for epidermal sensors. (a) Schematic illustration of an epidermal sensor for monitoring biological signals. (b) Schematic illustrations of a conventional Si chip-based design and fully flexible design of flexible sensor. (c) Circuit diagram of the sensor system consisting of a sensor, AFE, and RF frontend. The configuration of the AFE and roles of each element are also shown.

Because TFTs based on carbon nanotubes (CNTs) have relatively high mobility ${ }^{20-22}$ and excellent mechanical flexibility, ${ }^{23,24}$ they may help realise low-voltage flexible electronics for wearable devices. However, the electrical characteristics of CNT TFTs may have large deviceto-device variability because of the randomness of the network-like CNT thin film. ${ }^{25,26}$ This can significantly degrade the performance and increase the operating voltage of CNT-based circuits compared to the ideal case. In addition, CNT TFTs often exhibit instability issues such as hysteresis and threshold voltage drift under a bias stress. Addressing these issues is the most 
important challenge for realising high-performance CNT-based flexible electronics, especially

60 analogue circuits that require high stability.

We developed a methodology to realise low-voltage CNT-based flexible analogue/digital mixed-signal circuits with excellent stability and robustness against variability. A novel backside exposure self-aligned (BESA) process was developed for fabricating flexible CNT TFTs on a transparent plastic film, which minimises the parasitic capacitance and hence the delay time. We developed a device model and simulation tools for CNT-based analogue and digital mixed-signal circuits. The key circuit is a novel operational amplifier (op-amp) with a high gain and low operating voltage; this is important for the construction of negative-feedback circuits, which can dramatically suppress the influence of device variability and instability on the circuit performance. A bending test was conducted to demonstrate the excellent flexibility of the developed circuits. A flexible delta-sigma ADC with high energy efficiency was also realised. We used the above results to realise a fully flexible CNT AFE for the first time that monolithically integrates an instrument amplifier, bandpass filter, non-inverting amplifier, and delta-sigma $\mathrm{ADC}$ on a plastic film. In experiments, it demonstrated stable and continuous operation during the amplification and digitalisation of ECG signals. All flexible analogue and mixed-signal circuits fabricated in this work could be operated at a low supply voltage of \pm 1.5 V.

\section{BESA process for flexible CNT TFTs}

The self-aligned process is essential to reduce parasitic capacitance and improve the operating speed of CNT TFTs, particularly those fabricated on a flexible plastic film. Because such films slightly shrink during the device fabrication process, a large margin is required for layer-tolayer alignment. For example, a margin of $20 \mu \mathrm{m}$ is necessary to account for $0.1 \%$ shrinkage of a $20 \times 20 \mathrm{~mm}^{2}$ plastic film. Consequently, a large parasitic capacitance forms because of the overlap between the gate and source/drain electrodes. We developed the BESA process by 
utilising the transparency of the plastic film so that the source/drain electrodes self-align to the gate. Figures $2 \mathrm{a}$ and $2 \mathrm{~b}$ show CNT TFTs that were fabricated on a flexible and transparent polyethylene naphthalate (PEN) film with the BESA process. The BESA process took place during the lithography process for the source/drain formation; ultraviolet (UV) light was exposed from the backside of the PEN substrate through a photomask and gate electrode, which minimised the overlap between the gate and source/drain electrodes.

Figure 2c shows a micrograph of a CNT TFT fabricated by the BESA process $(W / L=100 / 10$ $\mu \mathrm{m})$. The overlap between the gate and source/drain is difficult to recognise. Figure $2 \mathrm{~d}$ shows the measured gate capacitance $\left(C_{\mathrm{G}}\right)$-gate voltage $\left(V_{\mathrm{GS}}\right)$ relationship. The parasitic capacitance due to the overlap can be observed in the off state of the $p$-type channel $\left(V_{\mathrm{GS}}>1 \mathrm{~V}\right)$. The offstate of the BESA-based CNT TFT was $0.32 \mathrm{pF}$, which was about one-eleventh of that for the conventionally fabricated device with a $10-\mu \mathrm{m}$ overlap $(3.65 \mathrm{pF})$. This demonstrated the effectiveness of the BESA process. The BESA process is also quite simple and reliable compared to the conventional self-aligned process. ${ }^{27}$ There was no device failure related to the BESA process, which indicates its suitability for application to large-area flexible integrated circuits (ICs) requiring a high performance level.

Figures $2 \mathrm{e}$ and $2 \mathrm{f}$ show the output and transfer characteristics, respectively, of a CNT TFT. The device exhibited typical $p$-type characteristics, and the mobility was estimated from the transconductance and $C_{\mathrm{G}}$ in the on state to have a maximum value of $42.7 \mathrm{~cm}^{2} / \mathrm{Vs}$ and average of $27.1 \mathrm{~cm}^{2} /$ Vs. These are comparable to the results of previous reports. ${ }^{28-30}$ For the circuit design in HSPICE, we developed a CNT TFT model by modifying a previous model ${ }^{31}$ for simulating devices operating in a deep off region, where minority carrier conduction occurs.

106 The current model fit the experimental data well (solid curves in Figures 2e and 2f). To obtain 107 the $C_{\mathrm{G}}-V_{\mathrm{GS}}$ characteristics, we applied the Meyer model often used for organic $\mathrm{TFTs}^{31}$ (see the 108 Supplementary Information for details on modelling CNT TFTs). 
109 Modelling the variability of CNT TFTs is indispensable for the design of reliable analogue

110 circuits, whose performance can be seriously affected by device variability. The device

111 characteristics were statistically analysed to extract model parameters such as the mobility,

112 threshold voltage, and oxide capacitance (see Supplementary Table S1). The Monte Carlo

113 method was used for the circuit simulation to account for device variability. The Monte Carlo

114 method (Figure 2i) closely reproduced the variability of the measured device characteristics

115 (Figure 2h).

a

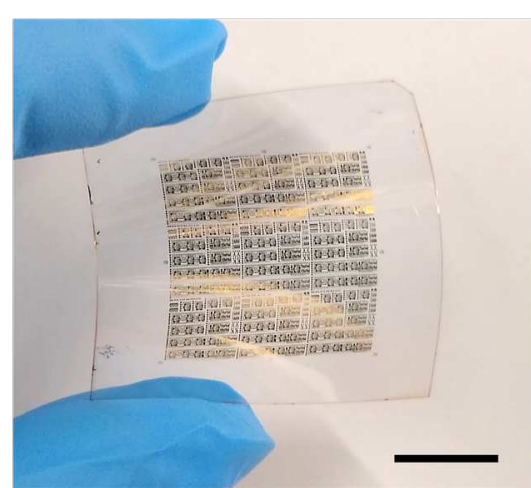

C

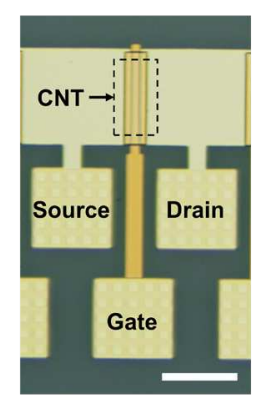

g

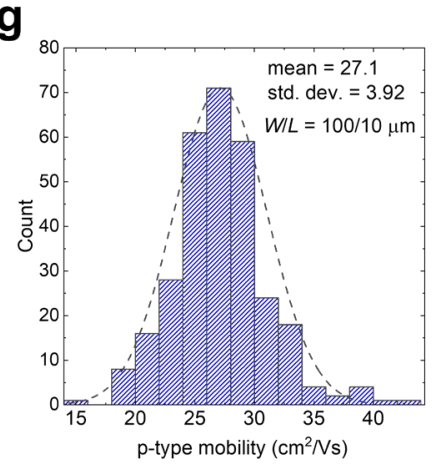
d

h b

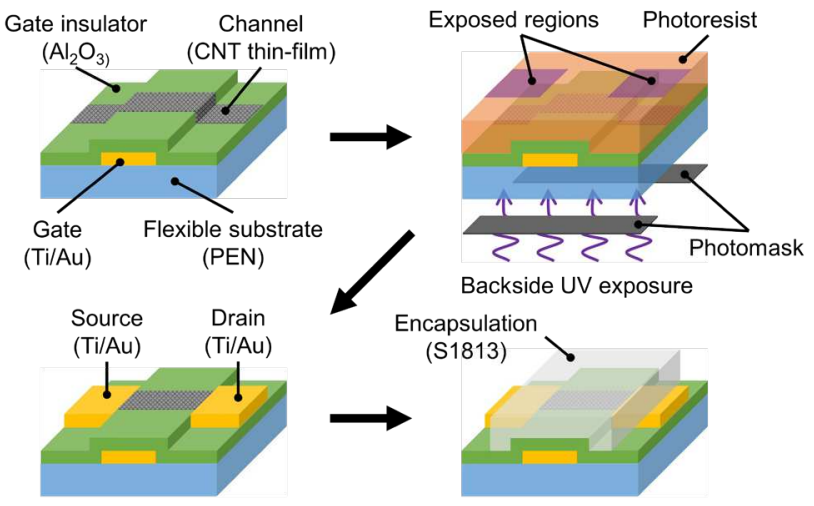

e
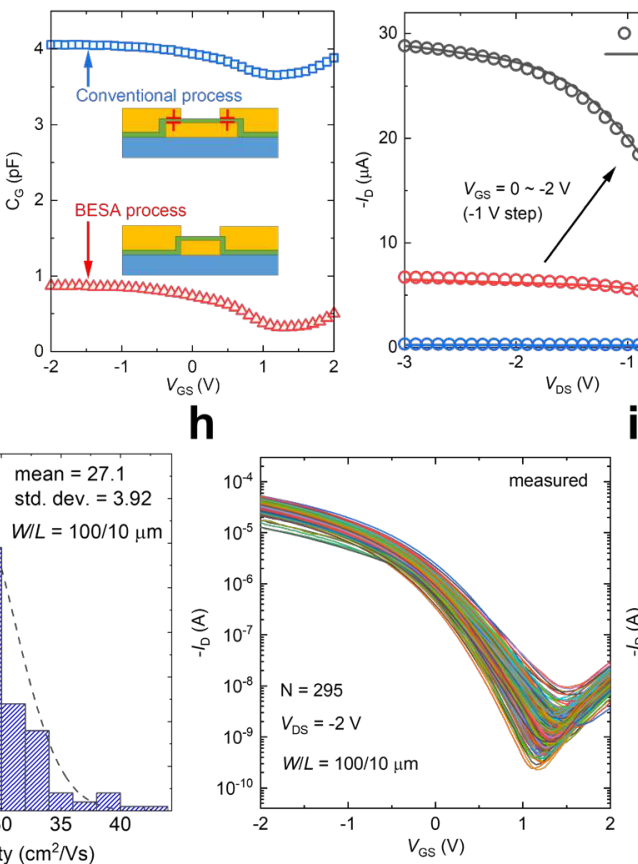

i
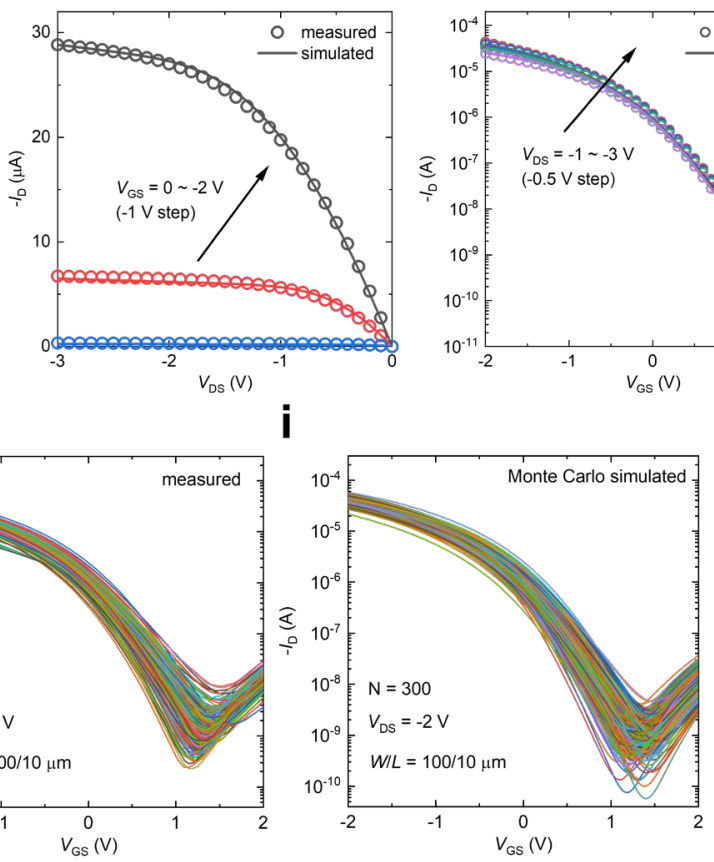

f

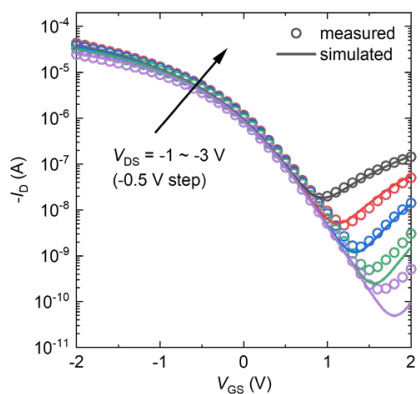

\section{(1)}

117 Figure 2 | Flexible CNT TFTs fabricated with the BESA process. (a) Photograph of devices

118 fabricated on a PEN film. The scale bar is $1 \mathrm{~cm}$. (b) Schematic illustration of the device 
119 fabricated with the BESA process. (c) Photograph of a CNT TFT. (d) $C_{\mathrm{G}}-V_{\mathrm{GS}}$ characteristics

120 of CNT TFTs fabricated by the conventional process and BESA process. (e) $I_{\mathrm{D}}-V_{\mathrm{DS}}$ and (f) $I_{\mathrm{D}}-$

$121 V_{\mathrm{GS}}$ characteristics of a typical CNT TFT. (g) Histogram of carrier mobility. The broken curve

122 is the Gaussian fit. The mean value and standard deviation were 27.1 and $3.92 \mathrm{~cm}^{2} / \mathrm{Vs}$. (h)

123 Measured and (i) simulated $I_{\mathrm{D}}-V_{\mathrm{GS}}$ characteristics of CNT TFTs at $V_{\mathrm{DS}}=-2 \mathrm{~V}$.

\section{Design and fabrication of high-gain and low-voltage op-amp}

125 A differential amplifier is often used for the first stage of the AFE to amplify weak sensor

126 signals. However, the amplification characteristics easily fluctuate because of the variability

127 and instability in the electrical properties of transistors. The key to solving the problem of

128 device variability is a negative-feedback circuit based on an op-amp. If the open-loop gain of

129 the op-amp is sufficiently high, then the amplification characteristics will be determined by

130 externally connected passive elements such as resistors and capacitors. Op-amps are also an

131 important core circuit often used in various functional circuits such as instrument amplifiers

132 and ADCs in the AFE and other analogue/digital mixed-signal circuits.

133 Figure 3a shows a typical two-stage CMOS-based op-amp that can provide a high gain with 134 relatively few transistors. However, this circuit configuration is sensitive to device variability, which can cause an input offset voltage $\left(V_{\mathrm{OS}}\right)$. In the ideal case, $V_{\mathrm{OS}}$ is zero; however, it becomes significant when paired transistors have mismatched characteristics. For example, $M_{1}: M_{2}$ or $M_{3}: M_{4}$ in Figure 3 a limits the output swing and gain at a low operating voltage. This 138 issue is particularly serious for op-amps using CNT TFTs, which have large variability. To solve this issue, we propose a novel op-amp circuit comprising single-polarity transistors

140 (Figure 3b) that has excellent robustness against device variability and requires a low operating 141 voltage.

142 Figure $3 \mathrm{c}$ shows a schematic of $V_{\mathrm{OS}}$ and its influence on the dynamic range of a non-inverting 143 amplifier, which comprises two resistors and a CMOS-based op-amp or the proposed op-amp. 
144 The input voltage $\left(V_{\mathrm{IN}}\right)$ consists of the AC input signal and additional DC offset voltage due to

$145 V_{\mathrm{OS}}$. With the CMOS-based op-amp, both the $\mathrm{AC}$ signal and $V_{\mathrm{OS}}$ are amplified by an 146 amplification factor of approximately $1+R_{2} / R_{1}$ because both the AC voltage gain $\left(A_{\mathrm{AC}}\right)$ and

147 DC voltage gain $\left(A_{\mathrm{DC}}\right)$ of the op-amp are relatively high (typically $\left.>50 \mathrm{~dB}\right)$. This causes a large

148 offset voltage at the output called the common-mode output voltage $\left(V_{\text {OCM }}\right)$, and the output

149 voltage swing is limited. Therefore, the output voltage waveform is likely to be distorted at a

150 low supply voltage when $A_{\mathrm{AC}}$ is high. In other words, device variability results in a trade-off

151 between a low operating voltage and high gain with the CMOS-based op-amp.

152 In contrast, the proposed op-amp can overcome this trade-off, even for CNT TFTs. The key to

153 the proposed op-amp is independent control of $A_{\mathrm{AC}}$ and $A_{\mathrm{DC}}$. It achieves a high $A_{\mathrm{AC}}$ of $>50 \mathrm{~dB}$

154 and small $A_{\mathrm{DC}}$ below unity simultaneously. The AC-coupled load consisting of a transistor and

155 a capacitor (surrounded with broken lines in Figure $3 b$ ) has a high/low impedance for the

$156 \mathrm{AC} / \mathrm{DC}$ component. The optimal $V_{\mathrm{B}}$ should realise a high $A_{\mathrm{AC}}=48 \mathrm{~dB}$ and small $A_{\mathrm{DC}}=-4.8$

$157 \mathrm{~dB}$. See the Supplementary information for the details.

158 The proposed circuit configuration has several advantages with regard to tolerance to

159 variability in the transistor characteristics. We performed a Monte Carlo simulation to 160 investigate the influence of the transistor variability on the non-inverting amplifier circuit.

161 Figure $3 \mathrm{~d}$ shows the $V_{\mathrm{OCM}}$ distributions of non-inverting amplifiers comprising the proposed 162 op-amp or CMOS op-amp. With the CMOS op-amp, $V_{\mathrm{OCM}}$ scattered widely from $V_{\mathrm{SS}}\left(=-V_{\mathrm{DD}}\right)$

163 to $V_{\mathrm{DD}}$ because of transistor variability. This eventually caused a large total harmonic distortion

164 (THD) of the output signal (Figure $3 \mathrm{e}$ ). In contrast, the proposed op-amp caused $V_{\mathrm{OCM}}$ to 165 converge around zero. Consequently, the THD of the non-inverting amplifier was effectively 166 suppressed, even when the variability in the transistor characteristics was accounted for. A low

167 THD is particularly important for sensor applications that require a linear detection response.

168 We confirmed that the proposed op-amp suppresses the variability in characteristics of a 
169 negative-feedback circuit. Figure $3 \mathrm{f}$ shows the open-loop voltage gain of the proposed op-amp 170 and closed-loop voltage gain of a non-inverting amplifier with the op-amp as a function of

171 frequency. Even for the open-loop voltage gain, a relatively narrow distribution was obtained

172 because of the small $V_{\mathrm{OCM}}$. A high open-loop gain is essential for proper operation of a

173 negative-feedback circuit. The variability was drastically suppressed with the non-inverting

174 amplifier circuit. Although we considered the static variability of transistor characteristics in 175 this simulation, a negative-feedback circuit is also effective at suppressing the influence of

176 electrical instability and drift in transistor characteristics, which are often observed for CNT 177 TFTs.

178 
a

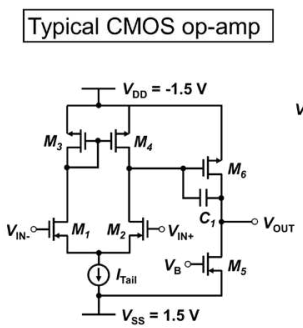

d

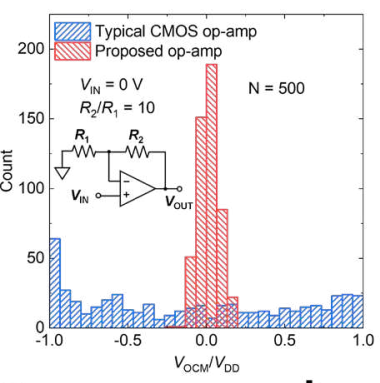

g

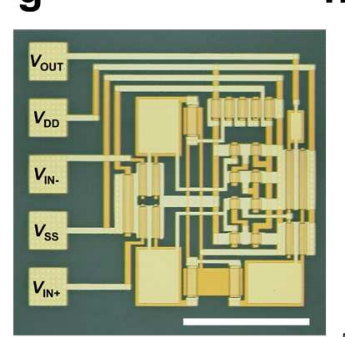

h b

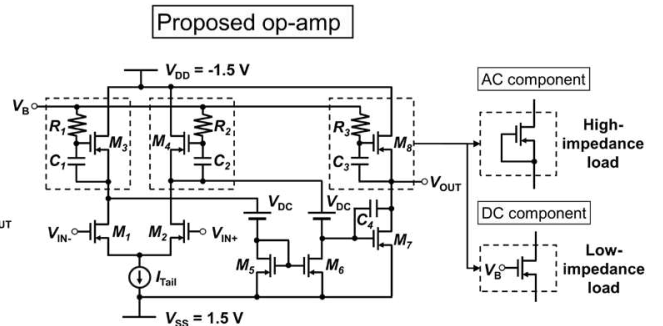

C

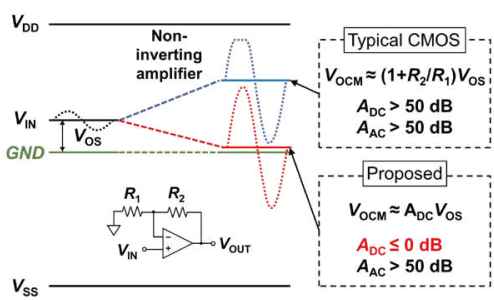

e

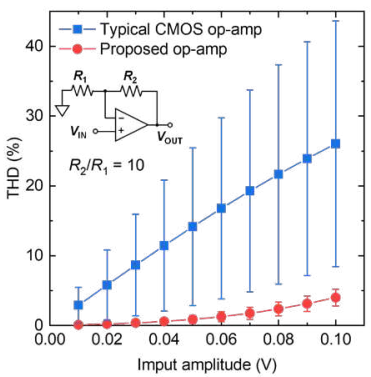

f

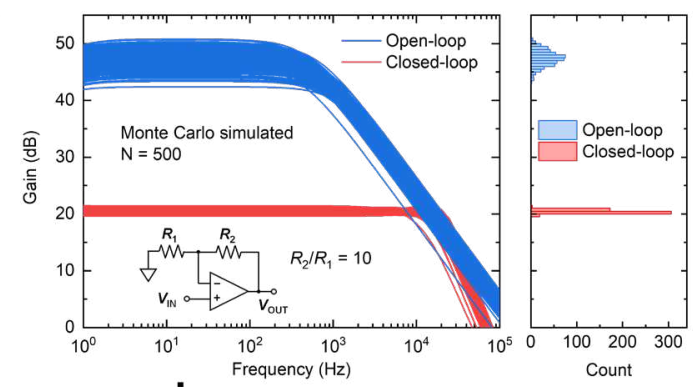

i
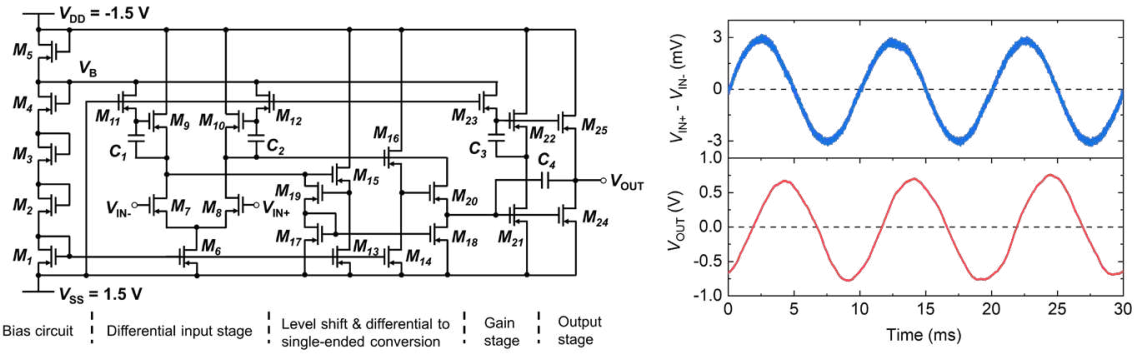

j

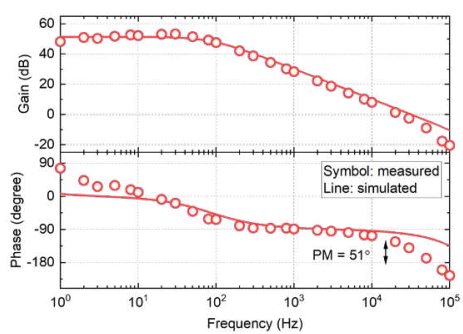

k

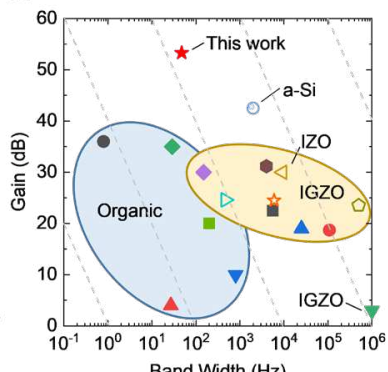

I

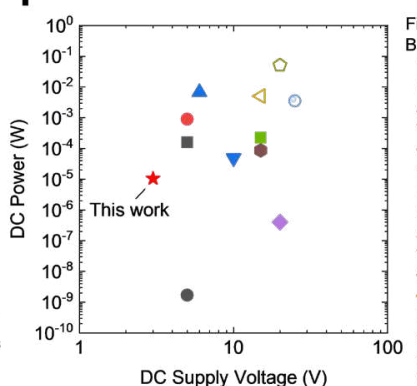

DC Supply Voltage (V)
Filled: Flexible substrate

n. Ishida, K (2015) [40]
I

Zysset, C (2013) [41]

Salvatore, G (2014) [32]

Garripoli, C (2017) [33]

Papadopoulos, N (2018) [34]

Marien, H (2012) [35]

Nausieda, I (2011) [36]

Chang, J (2014) [37]
Torres-Miranda, M (2016) [38]

Torres-Miranda, M (2016)
Sugiyama, M (2019) [39]

Chen, Z (2019) [47]

Chen, Z (2019) [47]
Kim, D (2018) [43]

Kim, D (2018) [43]
Rahaman, A (2019) [44]

Kim, K (2015) [45]
Tarn, Y (2010) [46]

180 Figure 3 | Design and fabrication of the high-gain and low-voltage op-amp with high

181 tolerance to transistor variability. (a) Circuit configuration of a typical two-stage CMOS-

182 based op-amp. (b) Circuit configuration of the proposed op-amp. (c) Schematics of the voltage

183 levels of $V_{\mathrm{OS}}$ and $V_{\mathrm{OCM}}$ for non-inverting amplifiers composed of the CMOS-based op-amp and

184 proposed op-amp, respectively. The inset shows the circuit configuration of the non-inverting

185 amplifier. Results of the Monte Carlo simulations of op-amps: (d) $V_{\text {OCM }}$ distribution normalised

186 by $V_{\mathrm{DD}}$, (e) THD as a function of the input voltage amplitude of non-inverting amplifiers 
composed of the CMOS op-amp and proposed op-amp, and (f) open-loop voltage gain of the proposed op-amp and closed-loop voltage gain of the non-inverting amplifier composed of the proposed op-amp as functions of the frequency and the maximum gain distributions. (g)

190 Photograph of the fabricated flexible CNT op-amp. The scale bar is $500 \mu \mathrm{m}$. (h) Circuit 191 diagram of the CNT op-amp. (i) Input (upper) and output (bottom) voltage waveforms. (j)

192 Fabrication and characterisation of the CNT op-amp. (k) Voltage gain versus bandwidth and 193 (I) DC power versus DC supply voltage of the present CNT op-amp and the differential amplifiers and op-amps reported previously for comparison.

A flexible op-amp based on CNT TFTs was fabricated on a PEN film (Figures $3 \mathrm{~g}$ and $3 \mathrm{~h}$ ) that fully integrates the circuits necessary to realise the proposed design described in the previous section: the bias circuit, differential input stage, level shift and differential-to-single-end converter, gain stage, and output stage. No additional bias voltage is necessary to control the bias point of the TFTs. See the Supplementary Information for the details of the op-amp circuit.

Figure $3 \mathrm{i}$ shows the voltage waveforms of the sinusoidal input $\left(V_{\mathrm{IN}+}-V_{\mathrm{IN}-}\right)$ and $V_{\mathrm{OUT}}$ at 100 $\mathrm{Hz}$. The power supply voltages were $V_{\mathrm{DD}}=-1.5 \mathrm{~V}$ and $V_{\mathrm{SS}}=+1.5 \mathrm{~V}$. The amplitude of the output voltage was $1.4 \mathrm{~V}_{\mathrm{pp}}$ for an input voltage of $6 \mathrm{mV}_{\mathrm{pp}}$, which indicated a high open-loop gain of $47.4 \mathrm{~dB}$ at $100 \mathrm{~Hz}$. Figure $3 \mathrm{j}$ shows the open-loop gain and phase as a function of the frequency. The gain in the low-frequency region was $53.3 \mathrm{~dB}$, and the bandwidth and unitygain frequency were 47.5 and $21.9 \mathrm{kHz}$, respectively. The phase margin, which could be controlled by the phase compensation capacitor $C_{4}$, was $51^{\circ}$. This large phase margin is sufficient for stable operation of a negative-feedback circuit without oscillation. Importantly, the measured characteristics of the fabricated op-amp (open circles in Figure 3j) showed good agreement with our simulation results (solid curves), which indicates a high degree of 210 completion for the device model and circuit design tools. 
211 We compared the performance of the present CNT op-amp with those of various differential 212 amplifiers $^{32-39}$ and op-amps: ${ }^{40-46}$ single-polarity TFTs on a flexible substrate ${ }^{32-42}$ or glass

213 substrate ${ }^{43-47}$ and with oxide semiconductors, ${ }^{32-34,40-45,47}$ organic semiconductors, ${ }^{35-39}$ or

214 amorphous $\mathrm{Si}^{46}$. The present op-amp clearly had the highest voltage gain (Figure 3k), which

215 was because of the proposed AC-coupling load circuit configuration. Most of the previous

216 differential amplifiers and op-amps had a gain of less than $30 \mathrm{~dB}$, which is insufficient for

217 negative-feedback circuits. The present op-amp had a higher gain-bandwidth product than

218 those based on organic semiconductors and comparable with those based on oxide

219 semiconductors.

220 The present op-amp could operate at a low supply voltage of $\pm 1.5 \mathrm{~V}$, which can be supplied by

221 lithium-ion batteries. It had the lowest operating voltage (Figure 3i), which led to a low power consumption of $10.5 \mu \mathrm{W}$. The low operating voltage and power consumption demonstrate the advantages of the CNT-based flexible op-amp for wearable device applications with regard to safety and limited power sources (see Supplementary Table S3).

\section{Negative-feedback circuits}

226 The greatest benefit of the present high-gain op-amp is the negative-feedback circuit, which provides various analogue functional circuits with better stability, reliability, and uniformity than other types of amplifier circuits. Here, we present a non-inverting amplifier and instrumentation amplifier; both are key components for constructing an AFE.

230 The non-inverting amplifier fabricated on a PEN film (Figures $4 \mathrm{a}$ and $4 \mathrm{~b}$ ) was driven at power supply of \pm 1.5 V. (See the Supplementary Information for the details.) Figure $4 \mathrm{c}$ shows the output voltage waveform for a sinusoidal voltage wave input at $10 \mathrm{~Hz}$. The output voltage exhibited an amplified sine wave without distortion. The voltage gain was $19.5 \mathrm{~dB}$, which is

234 close to the design value of $19.1 \mathrm{~dB}$ (4.8\% difference). The linearity was confirmed by the 235 measured spectrum of the output voltage (Figure 4d). The power densities of the second and 
third harmonics were negligible at -44.6 and $-46.9 \mathrm{~dB}$ relative to the fundamental wave, and

237 the THD was $0.8 \%$. The measured SNR was $28.8 \mathrm{~dB}$, which is greater than the $25 \mathrm{~dB}$ required

238 for ECG application. ${ }^{48}$ The bandwidth of the non-inverting amplifier was $2.46 \mathrm{kHz}$ with a

239 sufficient phase margin of $63^{\circ}$ (Figure 4e); this is also sufficient for monitoring various bio-

240 signals. The stability of the non-inverting amplifier was also investigated. Figure $4 \mathrm{f}$ shows the

241 changes in the gain, phase, $V_{\mathrm{OCM}}$, and SNR over a time of $1 \mathrm{~h}$. The gain, phase, and SNR are

242 fundamental performance indices of an amplifier and indicated excellent stability. The change

243 in $V_{\text {OCM }}$ was small at $6.7 \%$ of the power supply voltage, which would not affect the amplifier

244 performance. We examined the stability of the amplification characteristics against bending at various bending radii $(r)$ of $12.5,9.5$, and $8.0 \mathrm{~mm}$ (Figure $4 \mathrm{~g}$ ). The non-inverting amplifier exhibited almost no changes in the gain $(<1 \mathrm{~dB})$ and phase at $r=8.0 \mathrm{~mm}$ (Figure $4 \mathrm{~h}$ ).

An instrumentation amplifier was also fabricated on a PEN substrate (Figures $4 \mathrm{i}$ and $4 \mathrm{j}$ ) and consisted of three op-amps. One of the roles of an instrumentation amplifier in a sensor system is to reject common-mode signals, which normally originate from noise. In contrast, a differential-mode signal originates from a sensor signal. The common-mode rejection ratio (CMRR) is an important performance index for instrumentation amplifiers and is given by $C M R R=A_{\mathrm{DM}} / A_{\mathrm{CM}}$, where $A_{\mathrm{DM}}$ and $A_{\mathrm{CM}}$ are the differential- and common-mode gains, respectively (see the Supplementary Information for measuring $C M R R$ ). A high CMMR of $\sim 50$ $\mathrm{dB}$ was obtained in the present instrumentation amplifier (Figure 4k), so excellent noise reduction can be expected.

The experimentally measured characteristics showed good agreement with the simulation results for both the non-inversion amplifier and instrumentation amplifier (Figures $4 \mathrm{~g}$ and $4 \mathrm{i}$, respectively). This clearly demonstrates the reliability of the proposed circuit design technique and reproducibility of the device fabrication. 
a

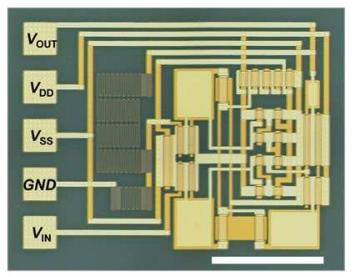

b

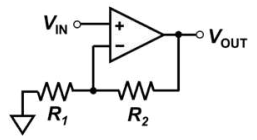

C

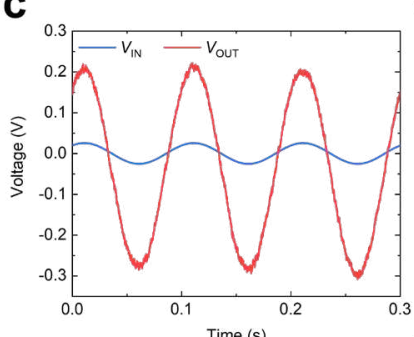

g

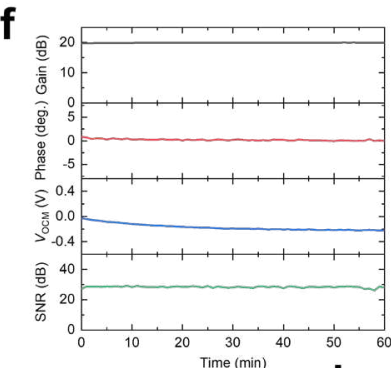

g

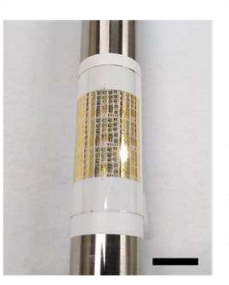

h

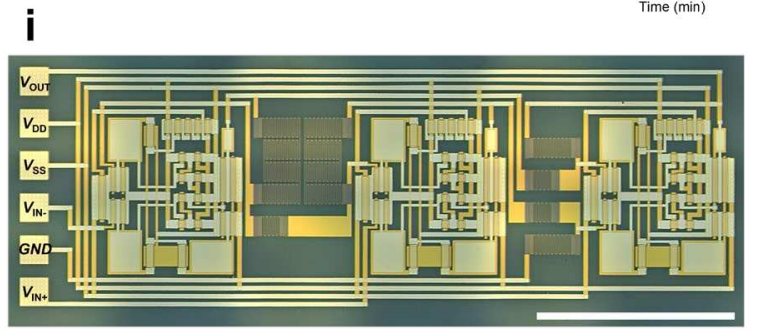

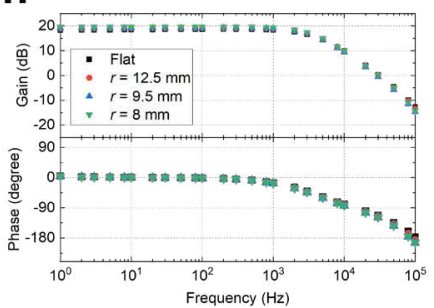

k

d

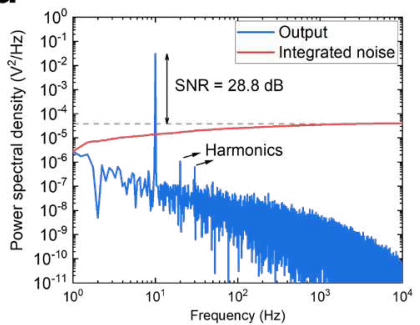

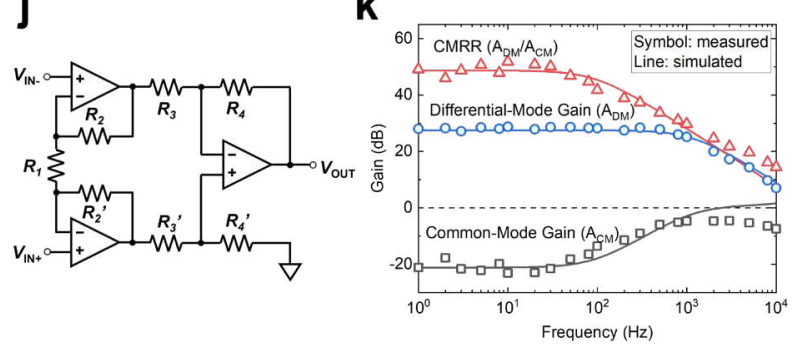

Figure 4 | Negative-feedback circuits composed of CNT op-amps. Non-inverting amplifier:

(a) photograph (scale bar: $500 \mu \mathrm{m}$ ), (b) circuit diagram, (c) input and output voltage waveform,

(d) power spectral density as a function of frequency, (e) measured (open circle) and simulated

(solid curve) gain (upper) and phase (bottom) as a function of frequency, and (f) changes in the gain, phase, $V_{\mathrm{OCM}}$, and SNR (from top to bottom) for $1 \mathrm{~h}$. (g) Photograph of the bending test (scale bar: $1 \mathrm{~cm}$ ), and (h) gain and phase as a function of frequency under various bending condition. Instrumentation amplifier: (i) photograph, (j) circuit diagram, and (k) CMRR, $A_{\mathrm{DM}}$, and $A_{\mathrm{CM}}$ as a function of frequency.

\section{Design and fabrication of a delta-sigma type ADC}

270 ADCs are an important component for robust signal transfer from a sensor system to an

271 information processing terminal. We designed a switched-capacitor (SC) delta-sigma $(\Delta \Sigma)$

272 ADC that can comprise relatively few transistors and operate with low power consumption

273 (Figures 5a and 5b). The $\Delta \Sigma \mathrm{ADC}$ is advantageous for sensor applications because of the high 
SNR in a low-frequency region from noise-shaping behaviour.

275 The fabricated ADC comprises a non-overlapping clock generator (Figure 5c), SC integrator, 276 latched comparator (Figure 5d), RS latch, and buffer. The pseudo complementary metal277 oxide-semiconductor (CMOS) technique ${ }^{49}$ is useful for improving the operating speed and 278 noise margin of digital ICs comprising single-polarity transistors and was introduced in the 279 digital gates such as latched comparator, NOT (Figure 5e), and NAND (Figure 5f). $\Delta \Sigma$ ADC 280 outputs a 1-bit pulse density modulation signal that depends on the input voltage. See the 281 Supplementary Information for the details of the operation.

282 The CNT-based flexible ADC was successfully operated at a power supply voltage of $\pm 1.5 \mathrm{~V}$, 283 clock frequency (CLK) of $10 \mathrm{kHz}$, oversampling rate (OSR) of 32, and signal bandwidth of $284156 \mathrm{~Hz}$ (Figure 5g). When the analogue input was a sine wave at $10 \mathrm{~Hz}$ and $1 \mathrm{~V}_{\mathrm{pp}}$, the pulse 285 density of the digital output increased with the analogue input voltage. The digital output was 286 reconverted to an analogue signal by a finite impulse response (FIR) decimation filter with a decimation rate of 32 on a PC. The sinusoidal signal was confirmed as reconstructed without distortion.

289 Figure 5h shows the power spectral density (PSD) of the digital output. The noise-shaping behaviour characteristic of $\Delta \Sigma$ ADCs was observed above $\sim 200 \mathrm{~Hz}$, so the noise level in the signal region $(<200 \mathrm{~Hz})$ was effectively reduced. The SNR and signal-to-noise distortion ratio

292 (SNDR) are shown in Figure 5i as functions of the input amplitude. A high SNR of $42 \mathrm{~dB}$ and 293 SNDR of $35 \mathrm{~dB}$ were obtained. The dynamic range (DR) was estimated to be $40 \mathrm{~dB}$ from the 294 ratio of the maximum input amplitude to the input amplitude to give $\mathrm{SNR}=0 \mathrm{~dB}$. The effective number of bits (ENOB) was determined to be 5.5 bit. The energy efficiency, which is commonly used as a figure of merit of ADCs, was estimated to be $2.06 \mathrm{~nJ} /$ conv.step from the

297 ENOB, bandwidth, and power consumption of $29.1 \mu \mathrm{W}$ (see the Supplementary Information).

298 The energy efficiency was one to two orders of magnitude better than those reported previously 
300 the high carrier mobility and low operating voltage of CNT TFTs and demonstrated the 301 advantage of CNT-based flexible electronics.

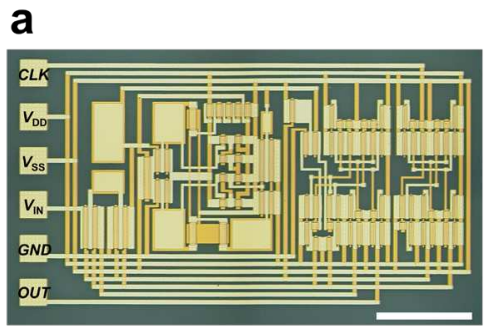

d
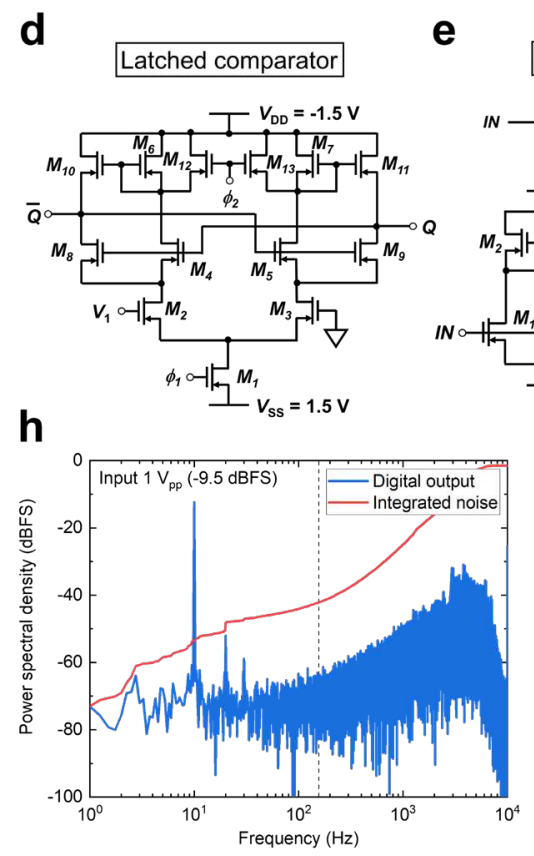

e b Switched-capacitor $\triangle \Sigma \mathrm{ADC}$

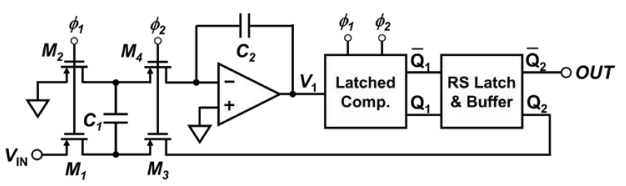

f
NOT<smiles>CC1CC1C</smiles>

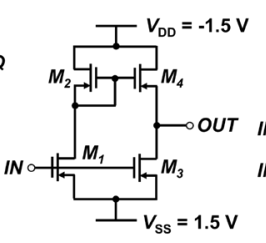

i

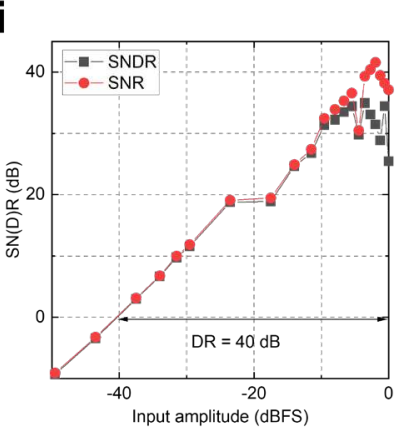

C

Non-overlapping clock generator

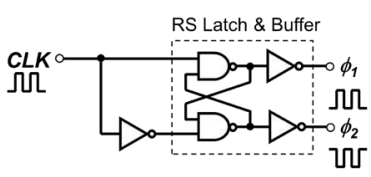

g
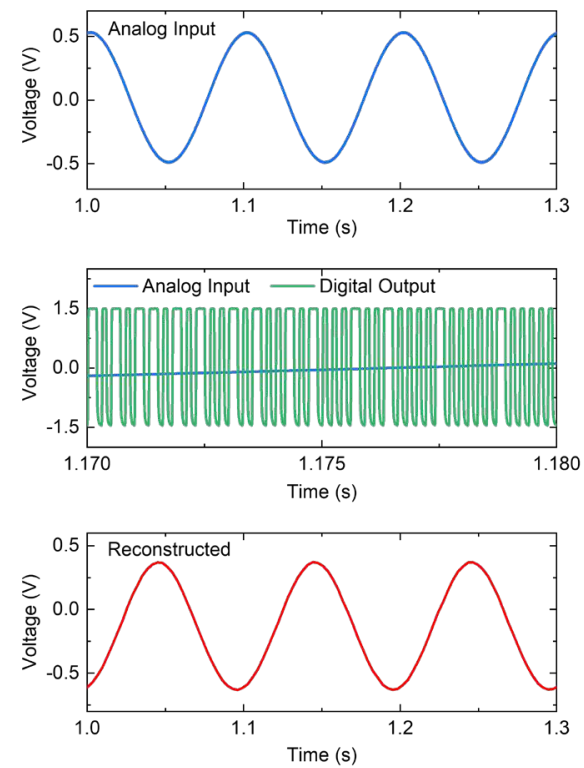

302

303 Figure 5 | Flexible CNT ADC. (a) Photograph (Scale bar: $500 \mu \mathrm{m}$ ) and (b) circuit diagram of

304 SC $\Delta \Sigma$ ADC fabricated in this study. Circuit diagrams of the (c) non-overlapping clock generator, (d) latched capacitor, (e) NOT gate, and (f) NAND gate. (g) Input voltage (top), analogue input voltage and digital output voltage on a magnified time scale (middle), and

307 analogue signal reconstructed from the digital output (bottom). (h) PSD and integrated noise

308 level of the digital output as a function of the frequency. (i) SNR and SNDR versus the input voltage amplitude. 
311 One of the most important applications of wearable flexible sensors is monitoring bio-signals.

312 For example, the ECG can be used not only to indicate exercise intensity and stress but also to

313 diagnose various diseases such as arrhythmia and angina pectoris. We realised a CNT-based

314 fully flexible AFE and demonstrated its feasibility and advantages for application as a wearable

315 ECG device.

316 The fabricated AFE consists of an instrumentation amplifier, bandpass filter, non-inverting 317 amplifier, and SC $\Delta \Sigma$ ADC (Figures $6 \mathrm{a}$ and $6 \mathrm{~b}$ ). All components were monolithically integrated 318 on a flexible plastic film. The CNT-based flexible AFE could also operate at a low supply 319 voltage of $\pm 1.5 \mathrm{~V}$ with a power consumption of $150 \mu \mathrm{W}$. We applied a simulated ECG signal 320 with an amplitude of $16 \mathrm{mV}_{\mathrm{pp}}$ to the AFE and then reconstructed the ECG signal on a PC from 321 the collected digital data.

322 Figure $6 \mathrm{c}$ shows a schematic for the coronary artery of a healthy person and the ECG with a 323 normal sinus rhythm. PQRST reflects the depolarisation and repolarisation of the heart's atria and ventricles, and the ST segment (i.e. flat region between the S and T waves) coincides with the baseline. Figure $6 \mathrm{f}$ shows the simulated ECG applied to the AFE as an input signal and the reconstructed waveform from the digital output. P- and T-wave peaks as well as the R-R

327 intervals corresponding to the heart rate can be observed in the reconstructed ECG.

328 Abnormal ECGs can also be distinguished. For example, an angina occurs when cholesterol builds up and plaque forms in the coronary arteries. In this case, the ST segment of the ECG waveform is known to be lower than the baseline (Figure 6d). If a blood clot forms in a coronary artery, a myocardial infarction occurs. This is reflected in the ECG by an elevation in the ST segment (Figure 6e). These characteristic behaviours of the ST segment can be seen in the reconstructed waveforms (Figures $6 \mathrm{~g}$ and $6 \mathrm{~h}$ ). We also confirmed that the AFE could operate continuously for at least $1 \mathrm{~h}$ (see Supplementary Figure S6). These results demonstrate that the CNT-based flexible AFE provides sufficient resolution to diagnose diseases from weak bio- 
a

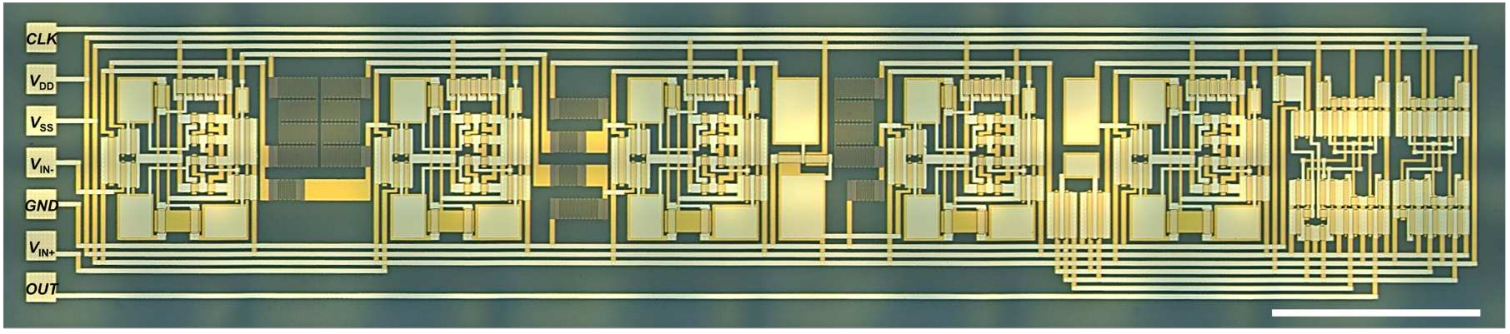

Instrumentation Amp.

$\begin{array}{l:l:}\text { BPF } & \text { Non-inv. Amp. }\end{array}$

$\Delta \Sigma$ AVD Converter

b
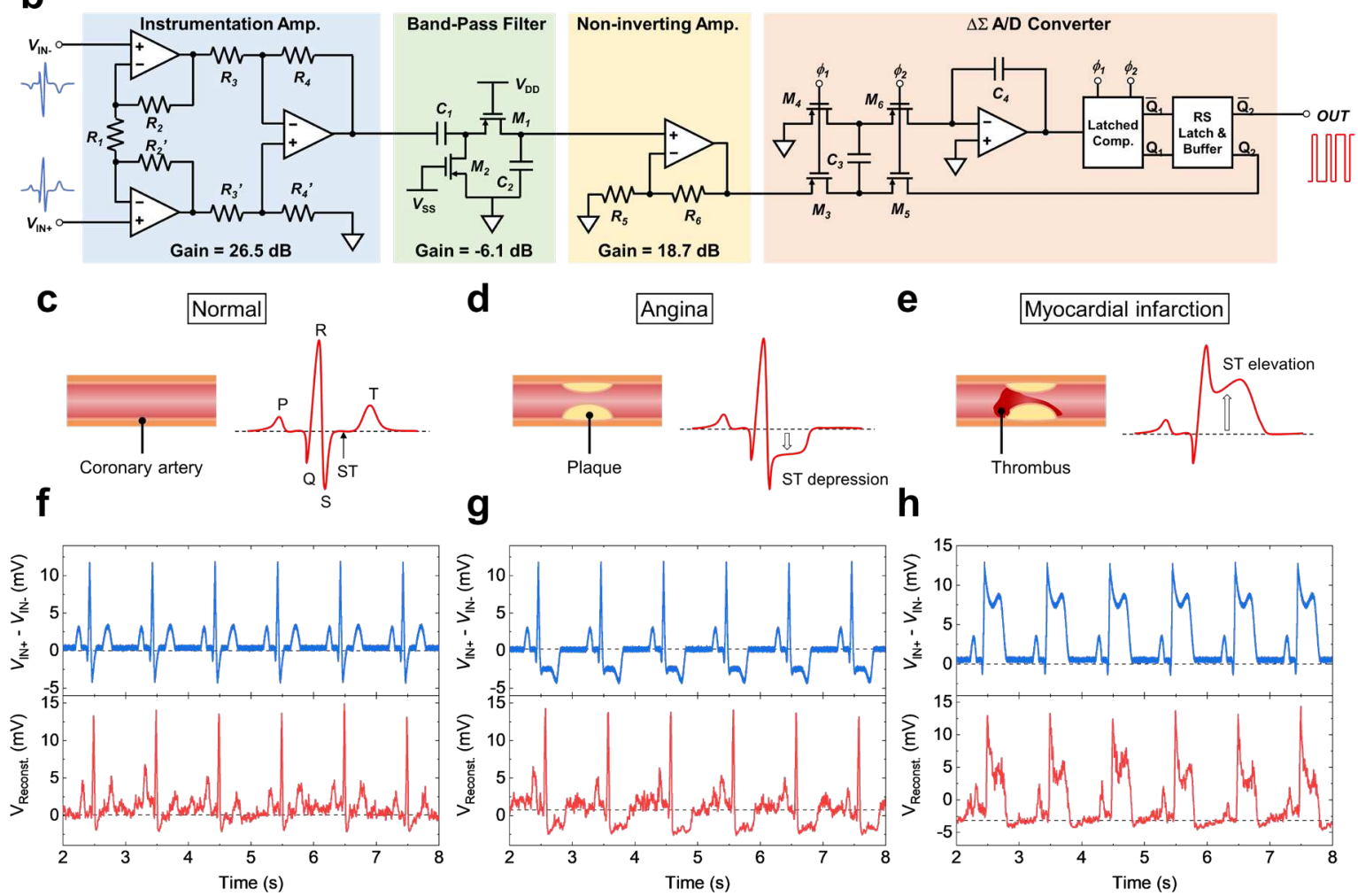

Figure 6 | CNT-based fully flexible AFE. (a) Photograph (scale bar: $1 \mathrm{~mm}$ ) and (b) block

339 diagram. Schematics of the coronary artery and ECG waveforms indicating (c) normal

340 conditions, (b) an angina, and (c) a myocardial infarction (c). Input voltage waveforms to the

341 AFM (upper) and reconstructed voltage waveforms from the digital output data (bottom)

342 indicating (f) normal conditions, (g) an angina, and (h) a myocardial infarction.

\section{Conclusion}

344 We developed a method for realising CNT-based flexible analogue circuits with excellent 
tolerance of variability and stability while requiring a low power consumption. A CNT op-amp

with high gain and high tolerance of device variation was developed with an AC-coupled load

347 circuit configuration and bias circuit to enhance $A_{\mathrm{AC}} / A_{\mathrm{DC}}$, which drastically suppresses the

348 variability of $V_{\mathrm{OCM}}$. We also developed a BESA process to minimise the parasitic capacitance,

349 which causes excess delay in CNT TFTs. The developed CNT op-amp realised a very high

350 voltage gain of $53.3 \mathrm{~dB}$ at a low supply voltage of $\pm 1.5 \mathrm{~V}$, which enabled the design of a stable

351 circuit system with negative feedback control. For example, a non-inverting amplifier was

352 developed that exhibited stable operation for at least $1 \mathrm{~h}$. We also developed an instrumentation

353 amplifier with a high CMRR of $50 \mathrm{~dB}$, which demonstrates excellent noise reduction

354 performance. A fully flexible AFE was monolithically integrated on a flexible plastic film; it

355 exhibited an excellent energy efficiency of $2.06 \mathrm{~nJ} /$ conv.step, and it successfully converted a

356 weak ECG signal of $16 \mathrm{mVpp}$ to robust digital data. Abnormal ST signals could be

357 distinguished in the ECG waveform reconstructed from the digital output.

358 Our results clearly demonstrate the advantages of CNT-based flexible analogue and

359 analogue/digital mixed-signal circuits; the low voltage and power consumption are particularly

360 important for realising wearable and epidermal sensor systems. In the future, low-voltage CNT-

361 based flexible electronics may pave the way to realising self-powered epidermal sensor systems

362 equipped with energy harvesters.

\section{Methods}

\section{Device fabrication process}

365 CNT TFTs and ICs were fabricated on a 125- $\mu$ m-thick PEN film. Gate electrodes of Ti/Au $366(1 / 50 \mathrm{~nm})$ were formed by conventional photolithography, electron beam evaporation, and the

367 lift-off process. Resistors were similarly formed by depositing 20-nm-thick nichrome. A 60-

$368 \mathrm{~nm}$-thick $\mathrm{Al}_{2} \mathrm{O}_{3}$ gate insulator was formed by atomic layer deposition at $140{ }^{\circ} \mathrm{C}$. Then, contact

369 windows were opened by $\mathrm{CF}_{4}$ plasma reactive ion etching of $\mathrm{Al}_{2} \mathrm{O}_{3}$. The semiconductor $\mathrm{CNT}$ 
thin film was formed by the transfer process as reported previously ${ }^{25}$ followed by oxygen

371 plasma etching. Source and drain electrodes of Ti/Au $(0.4 / 50 \mathrm{~nm})$ were formed by the BESA

372 process. The device was completed by encapsulation with a photoresist (Shipley, Microposit

$373 \mathrm{~S} 1813$ ) layer followed by baking at $130{ }^{\circ} \mathrm{C}$ for $20 \mathrm{~min}$.

374 Electrical measurements

375 All electrical measurements were carried out under ambient conditions. The $I-V$ and $C-V$ characteristics of the CNT TFTs were measured by an automatic probe station (Hisol, HSP100 ) with a semiconductor device parameter analyser (Keysight, B1500A). The CNT ICs were also characterised by the automatic probe station. The input voltage signal was generated by a function generator (Tektronics, AFG1062). A clock signal was applied from another function generator (Agilent, 33250A), and the output signal was measured with a digital oscilloscope (Tektronics, MOS58). In the durability test against bending, samples were bent by being attached to a rod with a diameter of 16,19 , or $25 \mathrm{~mm}$ and set on the probe station for electrical measurements.

\section{Device modelling and circuit simulation}

385 The device model for the CNT TFTs was developed from the measured electrical 386 characteristics. The model parameters were extracted by fitting the measured characteristics to

387 the model functions with Origin Pro (OriginLab) following the procedure reported by Deen et $388 a l .^{50}$ The mean and standard deviation of the model parameters were statistically obtained from 389 the data of 300 devices (see the Supplementary Information). The device model was described 390 with Verilog-A to include the HSPICE simulation. All circuit simulations such as transient, 391 DC, and AC analyses and the Monte Carlo simulations were performed with HSPICE. In the 392 CMOS circuit simulations, we assumed the parameters of the n-type device as equal to those 393 of the p-type device.

394 Reconstruction of analogue signal from digital data 
395 The output digital signal was measured and recorded by the digital oscilloscope in the comma396 separated values (CSV) data format. The digital data were transferred to a PC and converted to

397 an analogue signal through the FIR decimation filter programmed on Simulink in MATLAB 398 (MathWorks).

399 Generation of ECG signals

400 ECG waveforms were generated with an arbitral waveform generator (ArbExpress, Tektronics)

401 and transferred to the function generator (AFG1062, Tektronics) to output the differential 402 voltage of the ECG signal.

403 Data availability

404 The data that support the findings of this study are available from the corresponding author 405 upon reasonable request.

406 


\section{References}

408 1. Kim, Y. S. et al. All-in-one, wireless, stretchable hybrid electronics for smart, connected, 409 and ambulatory physiological monitoring. Adv. Sci. 6, 1900939 (2019).

410 2. Liu, Y. et al. Epidermal mechano-acoustic sensing electronics for cardiovascular 411 diagnostics and human-machine interfaces. Sci. Adv. 2, e1601185 (2016).

412 3. Khan, Y. et al. Flexible hybrid electronics: Direct interfacing of soft and hard electronics 413 for wearable health monitoring. Adv. Funct. Mater. 26, 8764-8775 (2016).

414 4. Yamamoto, Y. et al. Printed multifunctional flexible device with an integrated motion sensor for health care monitoring. Sci. Adv. 2, e1601473 (2016).

416 5. Xu, B. et al. An epidermal stimulation and sensing platform for sensorimotor prosthetic 417 control, management of lower back exertion, and electrical muscle activation. Adv. Mater. 28, 4462-4471 (2016).

6. Jeong, J. W. et al. Materials and optimized designs for human-machine interfaces via epidermal electronics. Adv. Mater. 25, 6839-6846 (2013).

7. Lee, H. et al. A graphene-based electrochemical device with thermoresponsive microneedles for diabetes monitoring and therapy. Nat. Nanotechnol. 11, 566-572 (2016).

8. Gao, W. et al. Fully integrated wearable sensor arrays for multiplexed in situ perspiration analysis. Nature 529, 509-514 (2016).

9. Ren, X. et al. A low-operating-power and flexible active-matrix organic-transistor temperature-sensor array. Adv. Mater. 28, 4832-4838 (2016).

10. Gao, L. et al. Epidermal photonic devices for quantitative imaging of temperature and thermal transport characteristics of the skin. Nat. Commun. 5, 1-10 (2014).

430 11. Nur, R. et al. A highly sensitive capacitive-type strain sensor using wrinkled ultrathin gold films. Nano Lett. 18, 5610-5617 (2018). 
12. Yamada, T. et al. A stretchable carbon nanotube strain sensor for human-motion detection. Nat. Nanotechnol. 6, 296-301 (2011).

13. Lee, H., Seong, B., Moon, H. \& Byun, D. Directly printed stretchable strain sensor based on ring and diamond shaped silver nanowire electrodes. RSC Adv. 5, 28379-28384 (2015).

14. Lee, S. et al. A transparent bending-insensitive pressure sensor. Nat. Nanotechnol. 11, 472-478 (2016).

15. Dagdeviren, C. et al. Conformable amplified lead zirconate titanate sensors with enhanced piezoelectric response for cutaneous pressure monitoring. Nat. Commun. 5, $1-10(2014)$.

16. Garripoli, C., Abdinia, S., van der Steen, J.-L. J. P., Gelinck, G. H. \& Cantatore, E. A fully integrated 11.2-mm² a-IGZO EMG front-end circuit on flexible substrate achieving up to 41-dB SNR and 29-M $\Omega$ input impedance. IEEE Solid-State Circuits Lett. 1, 142$145(2018)$.

17. Zulqarnain, M. et al. A low power time domain ECG interface based on flexible a-IGZO TFTs. in 2019 IEEE 8th International Workshop on Advances in Sensors and Interfaces (IWASI), Otranto, Italy, 13-14 June 2019, 205-209 (IEEE, 2019).

18. Zulqarnain, M. et al. A $52 \mu \mathrm{W}$ heart-rate measurement interface fabricated on a flexible foil with a-IGZO TFTs. in IEEE 44th European Solid State Circuits Conference (ESSCIRC), Dresden, Germany, 3-6 September 2018, 126-129 (IEEE, 2018).

19. Moy, T. et al. An EEG acquisition and biomarker-extraction system using low-noiseamplifier and compressive-sensing circuits based on flexible, thin-film electronics. IEEE J. Solid-State Circuits 52, 1-13 (2016).

20. Sun, D. M. et al. Flexible high-performance carbon nanotube integrated circuits. Nat. Nanotechnol. 6, 156-161 (2011). 
21. Wang, C. et al. Extremely bendable, high-performance integrated circuits using semiconducting carbon nanotube networks for digital, analog, and radio-frequency applications. Nano Lett. 12, 1527-1533 (2012).

22. Wang, C., Zhang, J. \& Zhou, C. Macroelectronic integrated circuits using highperformance separated carbon nanotube thin-film transistors. ACS Nano 4, 7123-7132 (2010).

23. Zhu, C. et al. Stretchable temperature-sensing circuits with strain suppression based on carbon nanotube transistors. Nat. Electron. 1, 183-190 (2018).

24. Sun, D. M. et al. Mouldable all-carbon integrated circuits. Nat. Commun. 4, 1-8 (2013).

25. Hirotani, J., Kishimoto, S. \& Ohno, Y. Origins of the variability of the electrical characteristics of solution-processed carbon nanotube thin-film transistors and integrated circuits. Nanoscale Adv. 1, 636-642 (2018).

26. Chen, J. \& Kumar, S. Variability in output characteristics of single-walled carbon nanotube thin-film transistors. IEEE Trans. Nanotechnol. 17, 353-361 (2018).

27. Zhang, Z. et al. Self-aligned ballistic n-type single-walled carbon nanotube field-effect transistors with adjustable threshold voltage. Nano Lett. 8, 3696-3701 (2008).

28. Chen, B. et al. Highly uniform carbon nanotube field-effect transistors and medium scale integrated circuits. Nano Lett. 16, 5120-5128 (2016).

29. Lei, T. et al. Low-voltage high-performance flexible digital and analog circuits based on ultrahigh-purity semiconducting carbon nanotubes. Nat. Commun. 10, 2161 (2019).

30. Wang, B.-W. et al. Continuous fabrication of meter-scale single-wall carbon nanotube films and their use in flexible and transparent integrated circuits. Adv. Mater. 30, 1802057 (2018).

480 31. Shao, L. et al. Compact modeling of thin film transistors for flexible hybrid IoT design. IEEE Des. Test 36, 6-14 (2019). 
32. Salvatore, G. A. et al. Wafer-scale design of lightweight and transparent electronics that wraps around hairs. Nat. Commun. 5, 2982 (2014).

33. Garripoli, C. et al. Analogue frontend amplifiers for bio-potential measurements manufactured with a-IGZO TFTs on flexible substrate. IEEE J. Emerg. Sel. Top. Circuits Syst. 7, 60-70 (2017).

34. Papadopoulos, N. et al. In-panel $31.17 \mathrm{~dB} 140 \mathrm{kHz} 87 \mu \mathrm{W}$ unipolar dual-gate In-Ga-ZnO charge-sense amplifier for 500dpi sensor array on flexible displays. in IEEE 44th European Solid State Circuits Conference (ESSCIRC), Dresden, Germany, 3-6 September 2018, 102-105 (IEEE, 2018).

35. Marien, H., Steyaert, M. S. J., Van Veenendaal, E. \& Heremans, P. Analog building blocks for organic smart sensor systems in organic thin-film transistor technology on flexible plastic foil. IEEE J. Solid-State Circuits 47, 1712-1720 (2012).

36. Nausieda, I. et al. Mixed-signal organic integrated circuits in a fully photolithographic dual threshold voltage technology. IEEE Trans. Electron Devices 58, 865-873 (2011).

37. Chang, J., Zhang, X., Ge, T. \& Zhou, J. Fully printed electronics on flexible substrates: High gain amplifiers and DAC. Org. Electron. 15, 701-710 (2014).

38. Torres-Miranda, M. et al. High-speed plastic integrated circuits: Process integration, design, and test. IEEE J. Emerg. Sel. Top. Circuits Syst. 7, 1-14 (2016).

39. Sugiyama, M. et al. An ultraflexible organic differential amplifier for recording electrocardiograms. Nat. Electron. 2, 351-360 (2019).

40. Ishida, K. et al. $22.5 \mathrm{~dB}$ open-loop gain, $31 \mathrm{kHz}$ GBW pseudo-CMOS based operational amplifier with a-IGZO TFTs on a flexible film. in 2014 IEEE Asian Solid-State Circuits Conference, KaoHsiung, Taiwan, 10-12 November 2014, 313-316 (IEEE, 2015).

41. Zysset, C. et al. IGZO TFT-based all-enhancement operational amplifier bent to a radius of 5 mm. IEEE Electron Device Lett. 34, 1394-1396 (2013). 
42. Shabanpour, R. et al. A $70^{\circ}$ phase margin OPAMP with positive feedback in flexible aIGZO TFT technology. in 2015 IEEE 58th International Midwest Symposium on Circuits and Systems (MWSCAS), Fort Collins, CO, USA, 2-5 August 2015, 13-16 (IEEE, 2015).

43. Kim, D., Kim, Y., Choi, K. Y., Lee, D. \& Lee, H. A solution-processed operational amplifier using direct light-patterned a-InGaZnO TFTs. IEEE Trans. Electron Devices 65, 1796-1802 (2018).

44. Rahaman, A., Chen, Y., Hasan, M. M. \& Jang, J. A high performance operational amplifier using coplanar dual gate a-IGZO TFTs. IEEE J. Electron Devices Soc. 7, 655661 (2019).

45. Kim, K., Choi, K. Y. \& Lee, H. a-InGaZnO thin-film transistor-based operational amplifier for an adaptive DC-DC converter in display driving systems. IEEE Trans. Electron Devices 62, 1189-1194 (2015).

46. Tarn, Y. C., Ku, P. C., Hsieh, H. H. \& Lu, L. H. An amorphous-silicon operational amplifier and its application to a 4-bit digital-to-analog converter. IEEE J. Solid-State

47. Chen, Z. J. et al. A new high-gain operational amplifier using transconductanceenhancement topology integrated with metal oxide TFTs. IEEE J. Electron Devices Soc. 7, 118-126 (2019).

48. Chantaf, S., Naït-Ali, A., Karasinski, P. \& Khalil, M. ECG modelling using wavelet networks: Application to biometrics. Int. J. Biom. 2, 236-249 (2010).

49. Myny, K. The development of flexible integrated circuits based on thin-film transistors. Nat. Electron. 1, 30-39 (2018).

50. Deen, M. J., Marinov, O., Zschieschang, U. \& Klauk, H. Organic thin-film transistors: 


\section{Acknowledgement}

534 This work was partially supported by the Japan Science and Technology Agency CREST 535 program (JPMJCR16Q2), MEXT KAKENHI (Grant Number JP15H05867), and JSPS 536 KAKENHI (Grant Number JP20H00243).

\section{Author contributions}

538 Y.O. conceived and supervised this work. T.K. designed and performed the experiments. H.K.

539 prepared the purified semiconducting CNTs. S.K. designed the nichrome resistors. T.K. and

540 Y.O. co-wrote the paper. All authors discussed the results and commented on the manuscript.

\section{Conflicts of interest}

542 The authors have no conflicts of interest to declare. 
a

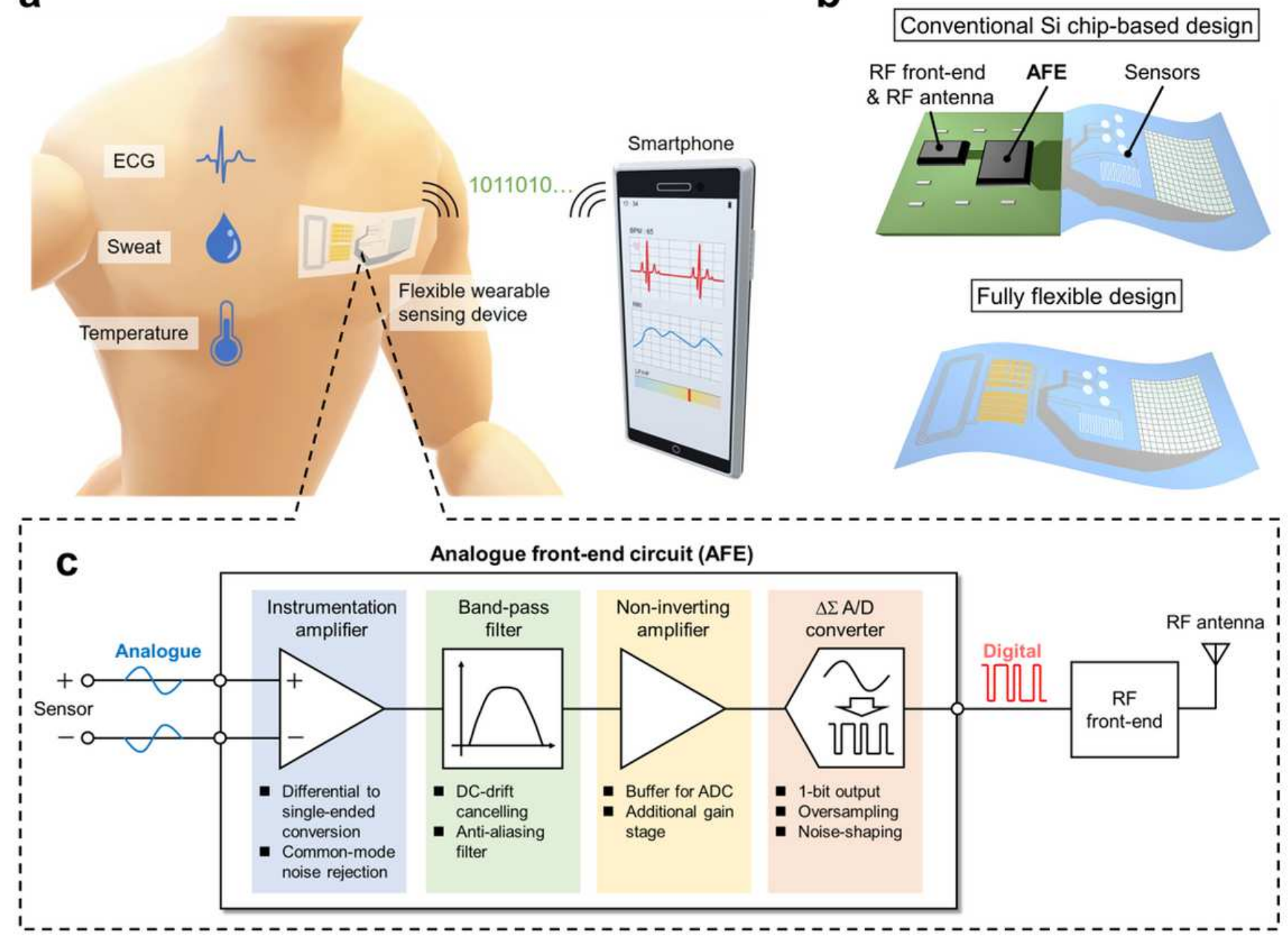

\section{Figure 1}

Fully flexible electronics for epidermal sensors. (a) Schematic illustration of an epidermal sensor for monitoring biological signals. (b) Schematic illustrations of a conventional Si chip-based design and fully flexible design of flexible sensor. (c) Circuit diagram of the sensor system consisting of a sensor, AFE, and RF frontend. The configuration of the AFE and roles of each element are also shown. 
a

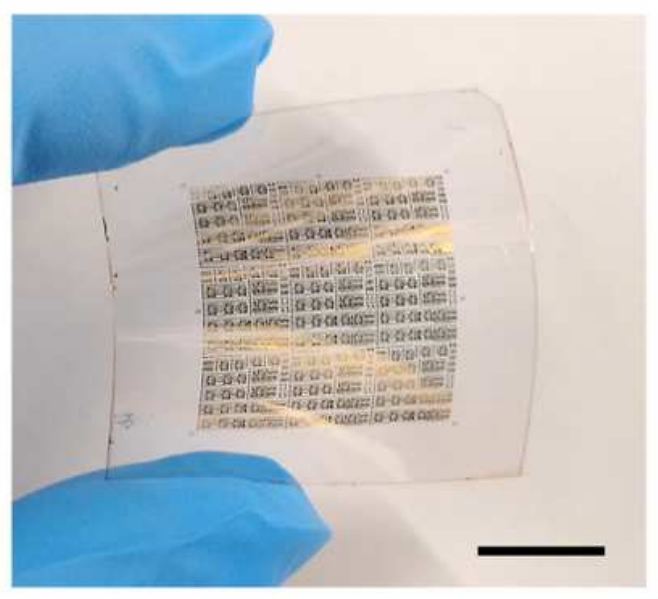

c

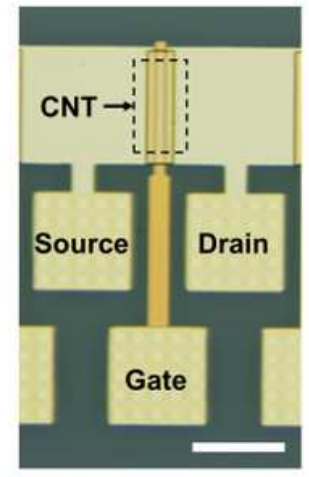

d

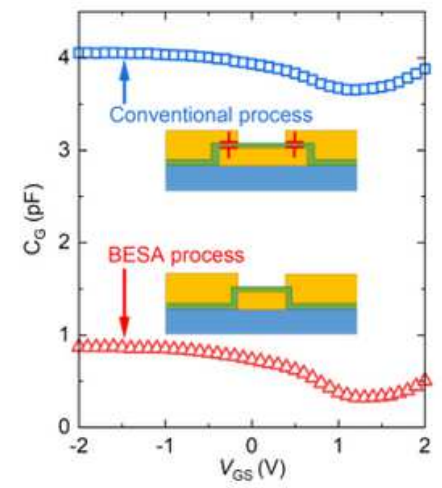

g

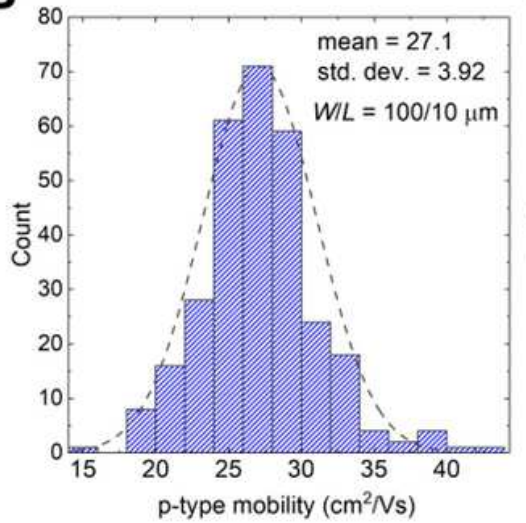

h b
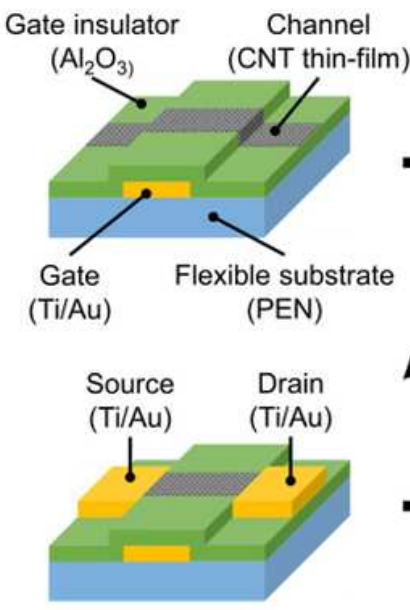

e

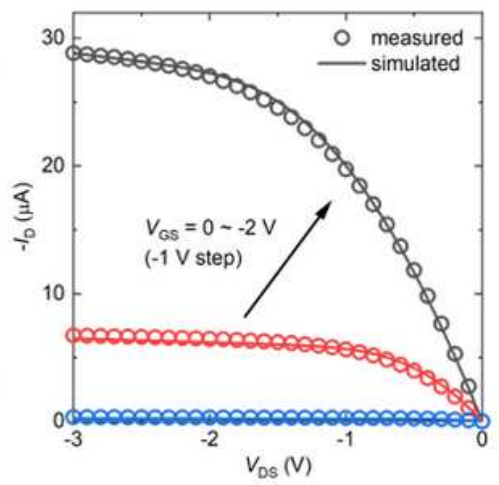

Exposed regions Photoresist

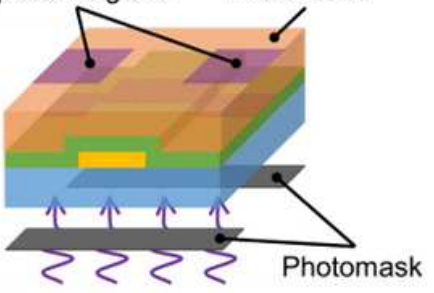

Backside UV exposure

Encapsulation

(S1813)

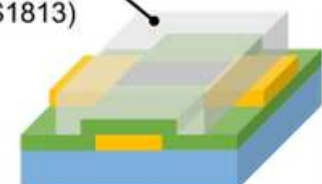

f

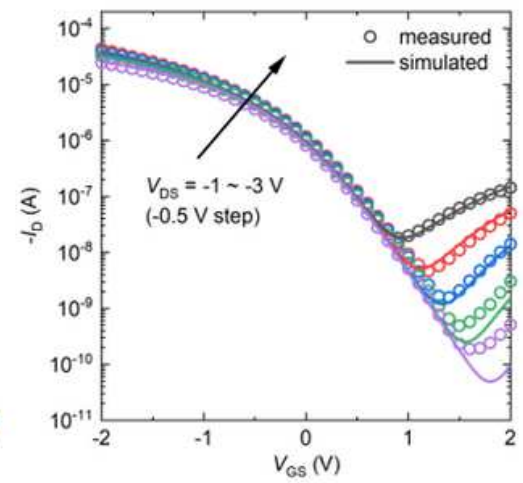

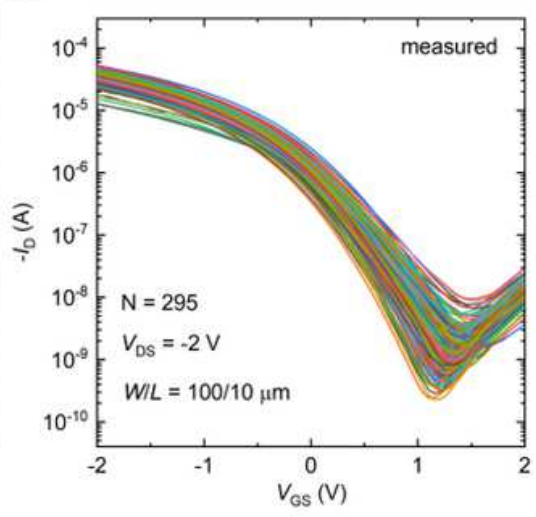

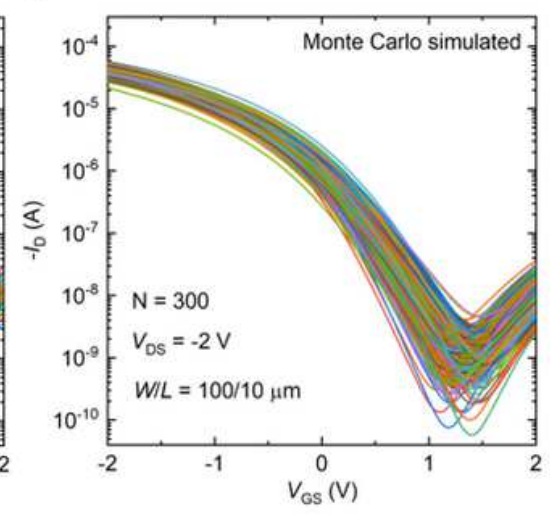

\section{Figure 2}

Flexible CNT TFTs fabricated with the BESA process. (a) Photograph of devices fabricated on a PEN film. The scale bar is $1 \mathrm{~cm}$. (b) Schematic illustration of the device fabricated with the BESA process. (c) Photograph of a CNT TFT. (d) CG-VGS characteristics of CNT TFTs fabricated by the conventional process and BESA process. (e) ID-VDS and (f) ID-VGS characteristics of a typical CNT TFT. (g) Histogram of carrier mobility. The broken curve is the Gaussian fit. The mean value and standard deviation were 27.1 and $3.92 \mathrm{~cm} 2 / \mathrm{Vs}$. (h) Measured and (i) simulated ID-VGS characteristics of CNT TFTs at VDS $=-2 \mathrm{~V}$. 
a

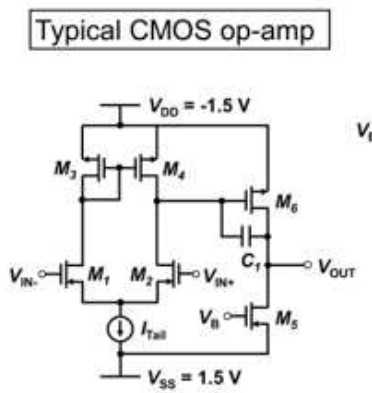

d
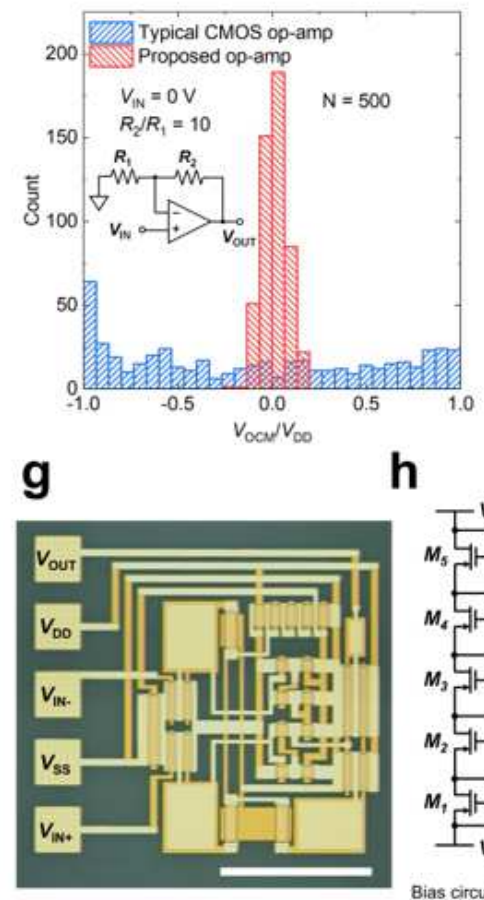

h

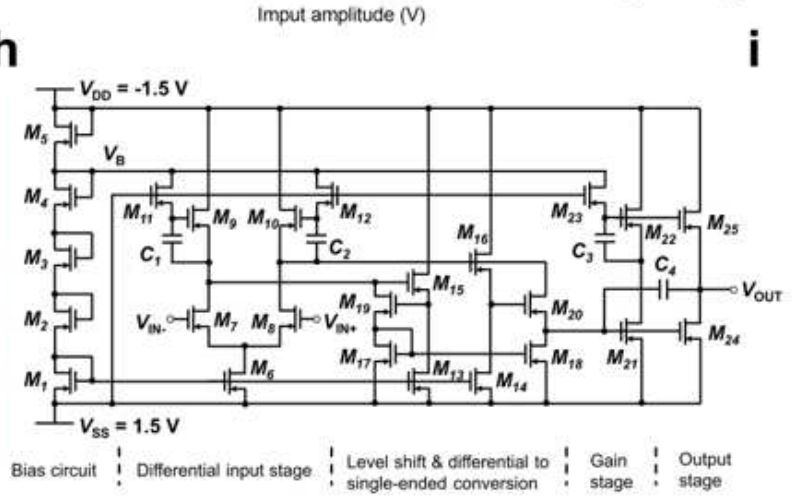

e

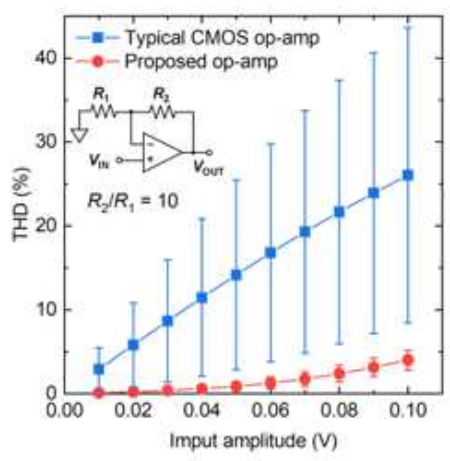

$\mathbf{k}$
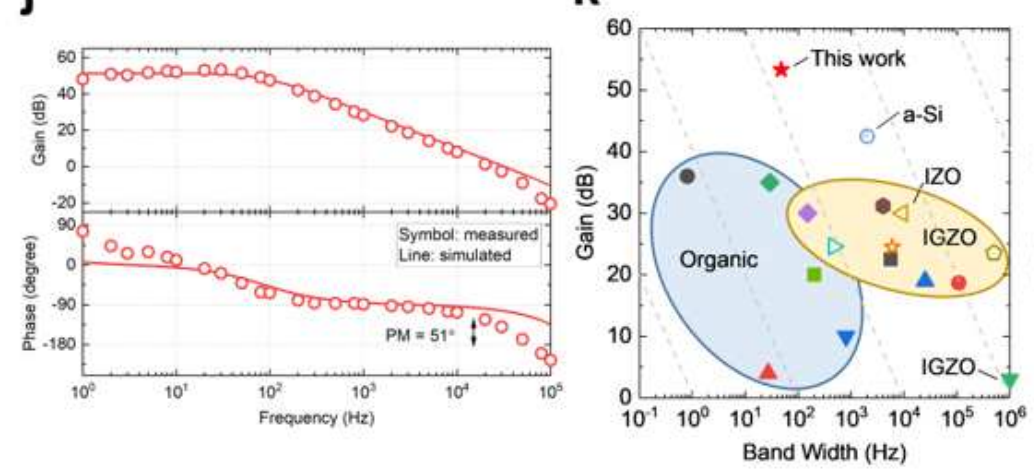

(1)
C

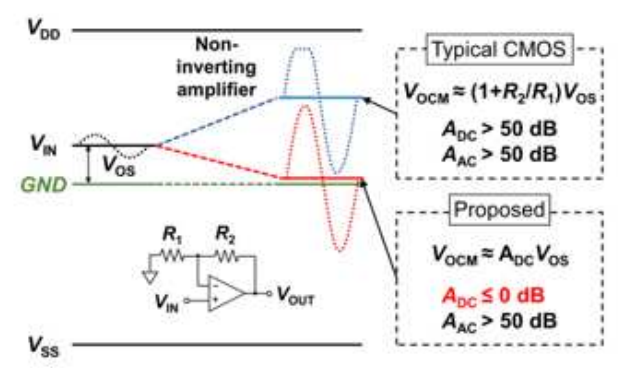

f
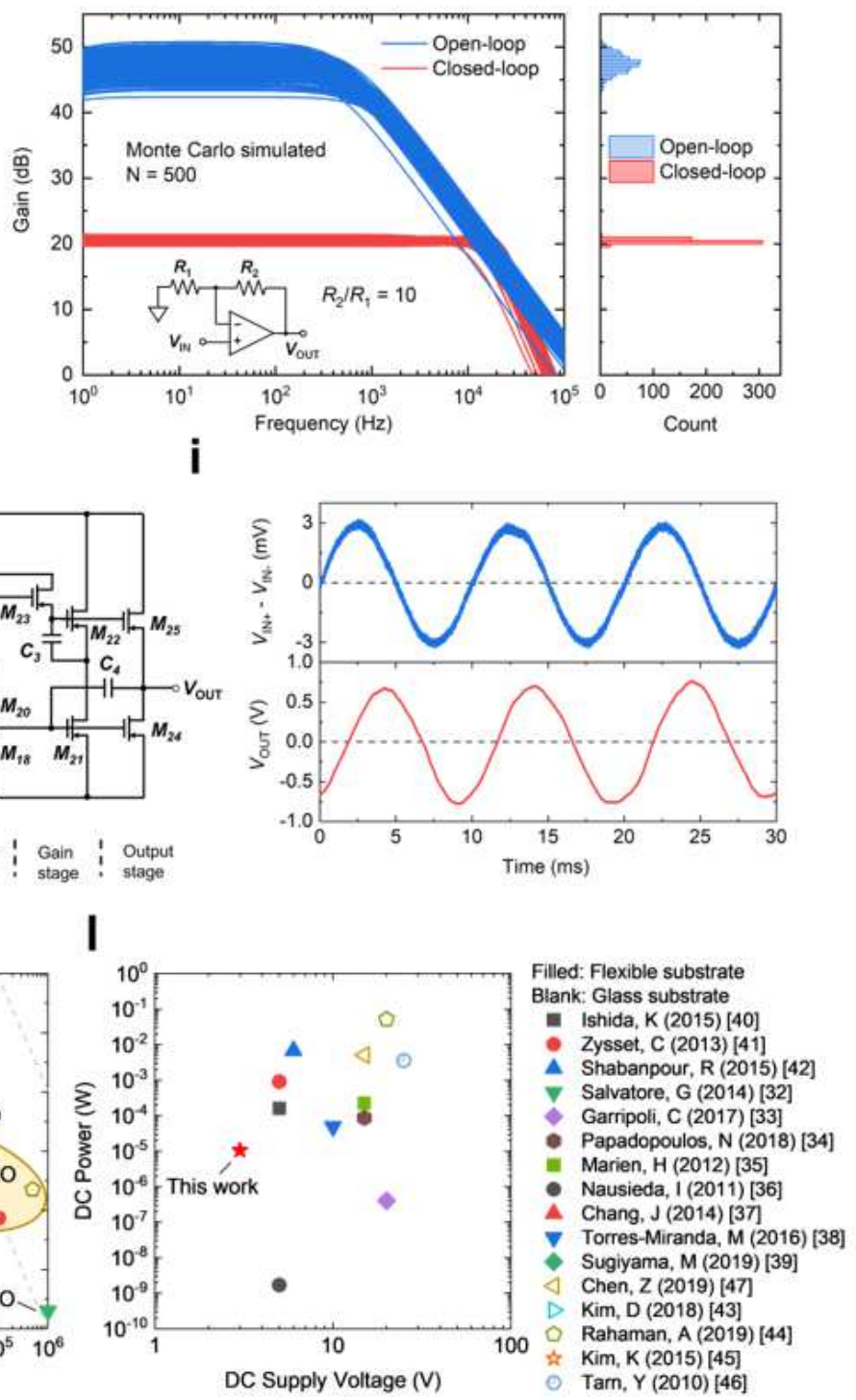

\section{Figure 3}

Design and fabrication of the high-gain and low-voltage op-amp with high tolerance to transistor variability. (a) Circuit configuration of a typical two-stage CMOS-based op-amp. (b) Circuit configuration of the proposed op-amp. (c) Schematics of the voltage levels of VOS and VOCM for non-inverting amplifiers composed of the CMOS-based op-amp and proposed op-amp, respectively. The inset shows the circuit configuration of the non-inverting amplifier. Results of the Monte Carlo simulations of op- 
amps: (d) VOCM distribution normalised by VDD, (e) THD as a function of the input voltage amplitude of non-inverting amplifiers composed of the CMOS op-amp and proposed op-amp, and (f) open-loop voltage gain of the proposed op-amp and closed-loop voltage gain of the non-inverting amplifier composed of the proposed op-amp as functions of the frequency and the maximum gain distributions. (g) Photograph of the fabricated flexible CNT op-amp. The scale bar is $500 \mu \mathrm{m}$. (h) Circuit diagram of the CNT op-amp. (i) Input (upper) and output (bottom) voltage waveforms. (j) Fabrication and characterisation of the CNT opamp. (k) Voltage gain versus bandwidth and (I) DC power versus DC supply voltage of the present CNT op-amp and the differential amplifiers and op-amps reported previously for comparison.

a

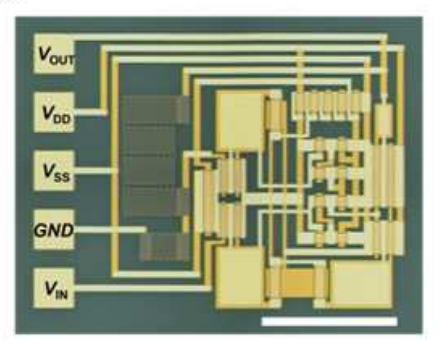

b

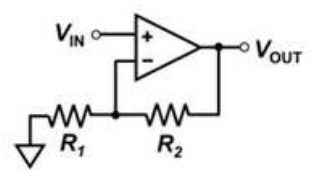

e
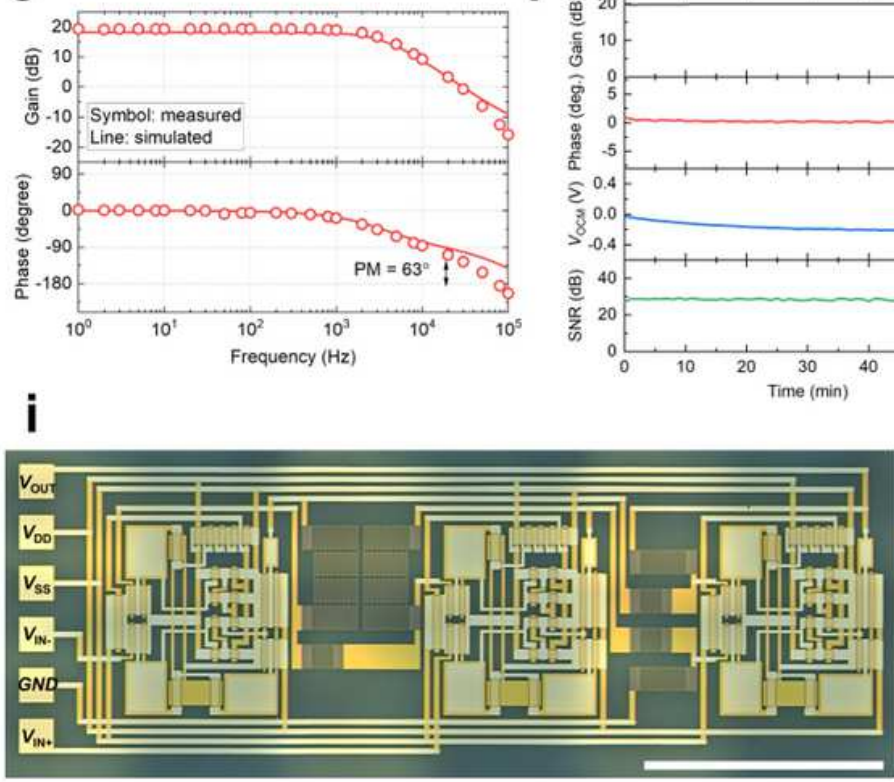

c

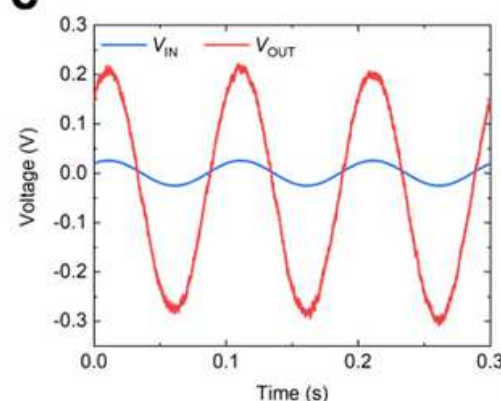

g
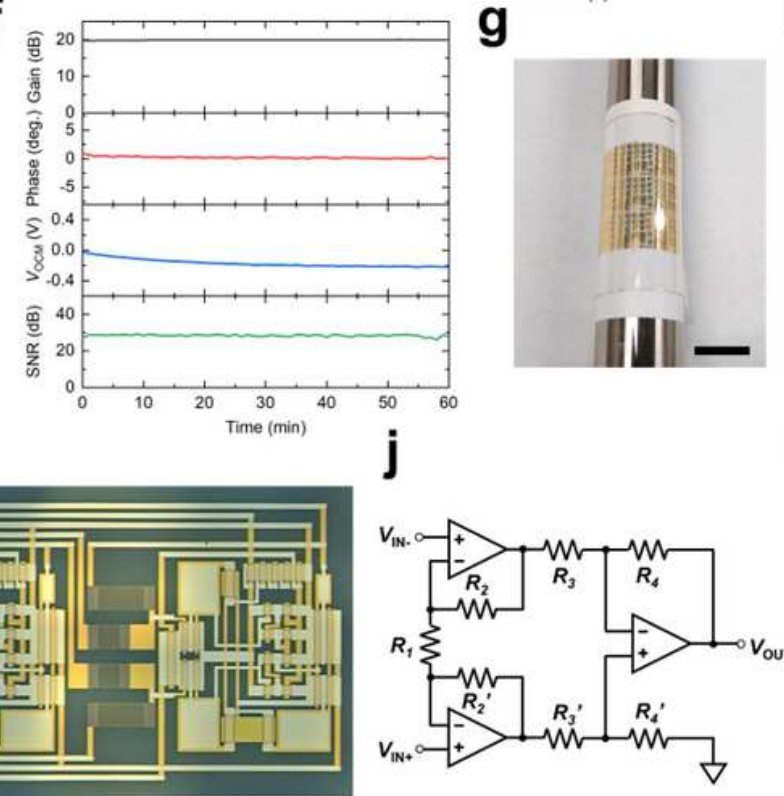

d

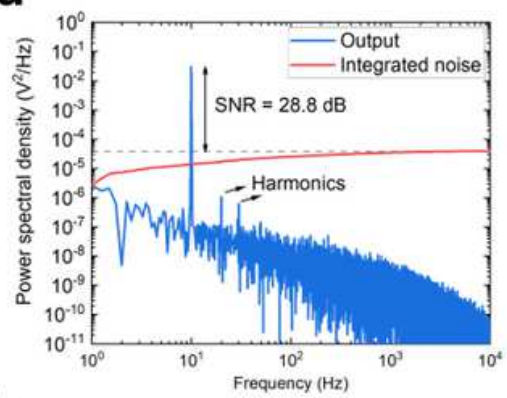

h

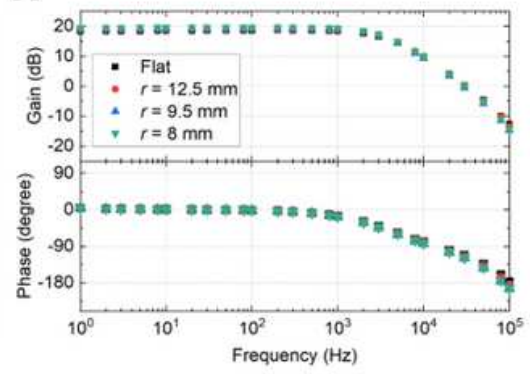

k

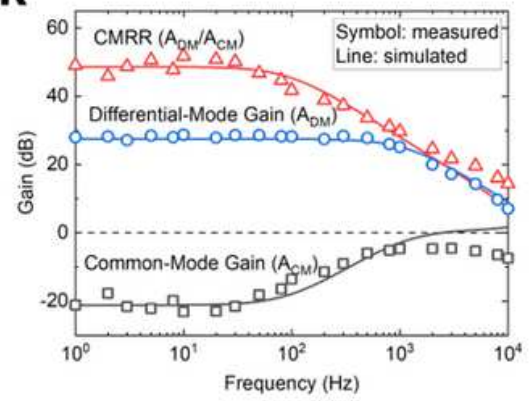

\section{Figure 4}

Negative-feedback circuits composed of CNT op-amps. Non-inverting amplifier: (a) photograph (scale bar: $500 \mu \mathrm{m}$ ), (b) circuit diagram, (c) input and output voltage waveform, (d) power spectral density as a function of frequency, (e) measured (open circle) and simulated (solid curve) gain (upper) and phase (bottom) as a function of frequency, and (f) changes in the gain, phase, VOCM, and SNR (from top to bottom) for $1 \mathrm{~h}$. (g) Photograph of the bending test (scale bar: $1 \mathrm{~cm}$ ), and (h) gain and phase as a function of frequency under various bending condition. Instrumentation amplifier: (i) photograph, (j) circuit diagram, and ( $k$ ) CMRR, ADM, and ACM as a function of frequency. 
a

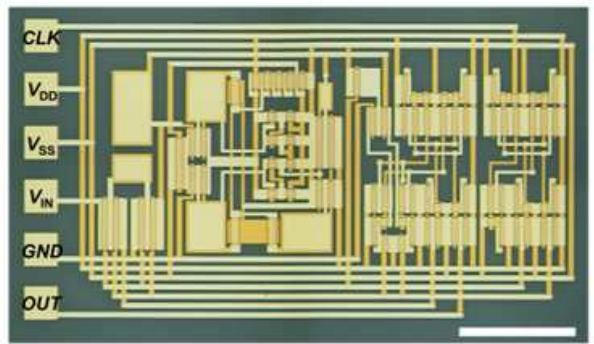

b

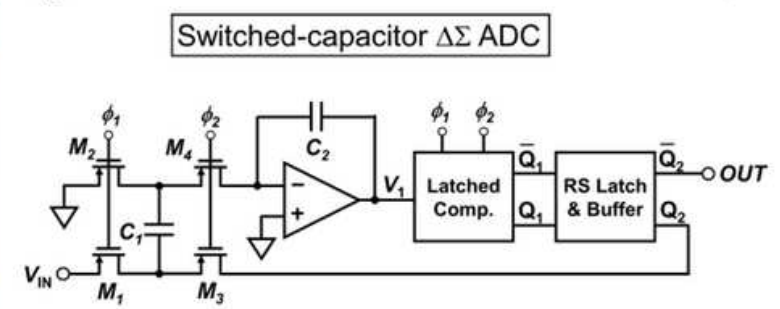

C

Non-overlapping clock generator

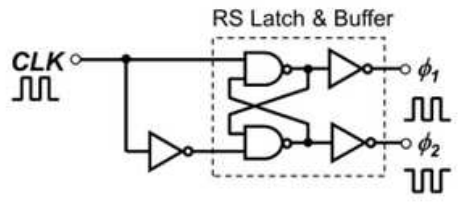

d

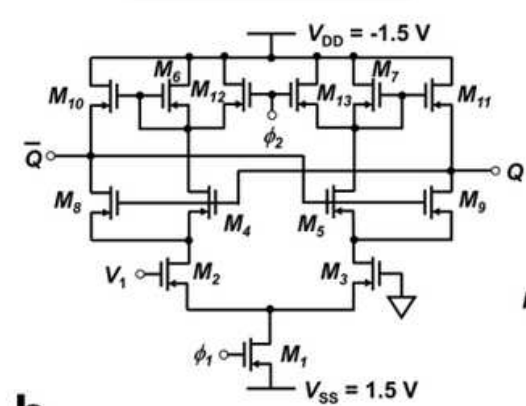

h

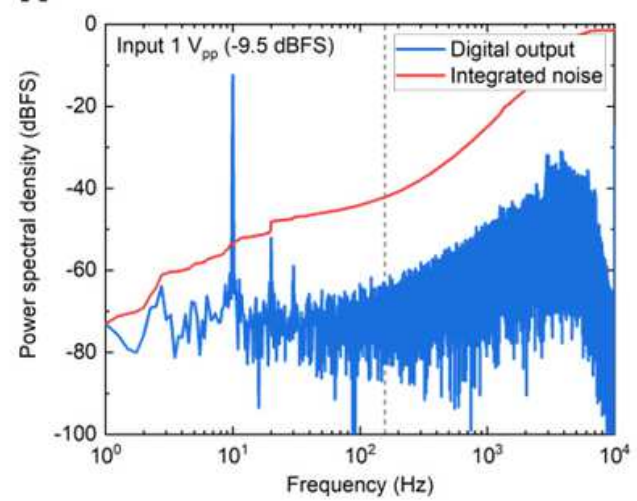

e
NOT
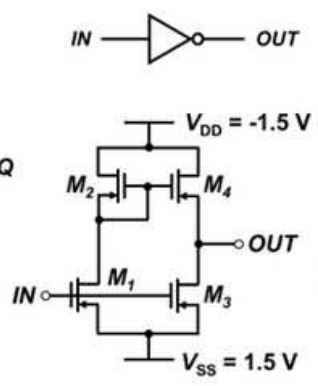

f NAND
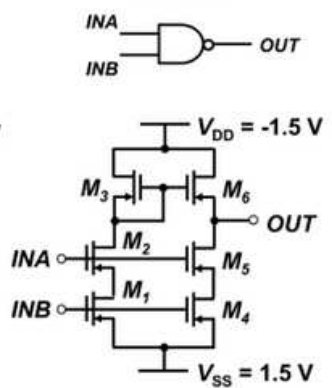

i

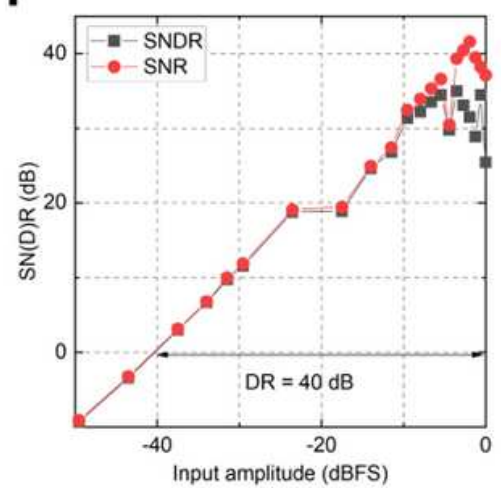

g
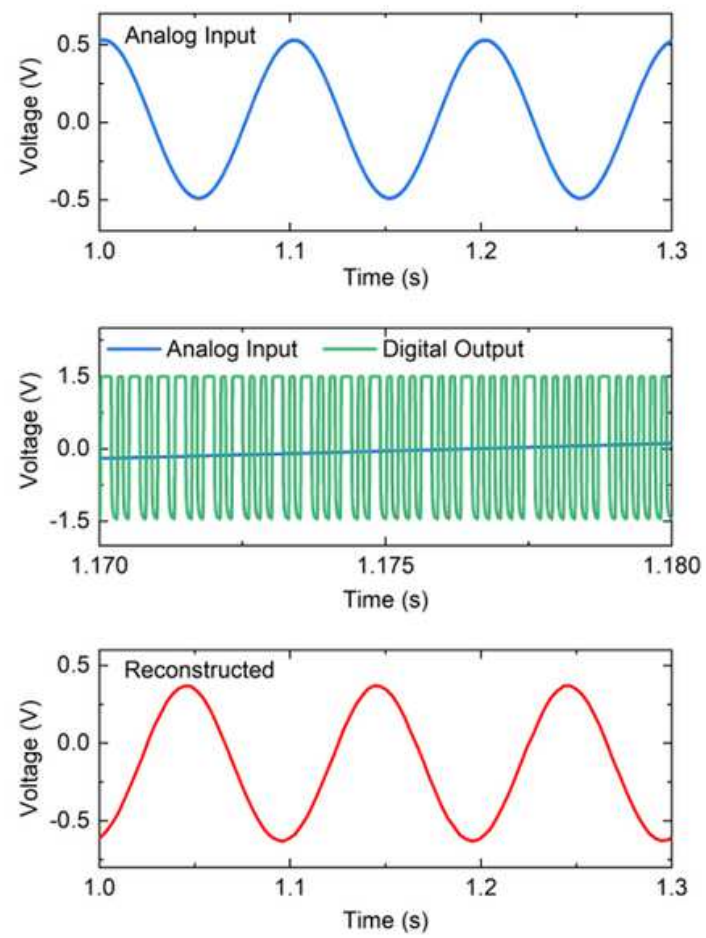

Figure 5

Flexible CNT ADC. (a) Photograph (Scale bar: $500 \mu \mathrm{m}$ ) and (b) circuit diagram of SC $\triangle \Sigma$ ADC fabricated in this study. Circuit diagrams of the (c) non-overlapping clock generator, (d) latched capacitor, (e) NOT gate, and (f) NAND gate. (g) Input voltage (top), analogue input voltage and digital output voltage on a magnified time scale (middle), and analogue signal reconstructed from the digital output (bottom). (h) PSD and integrated noise level of the digital output as a function of the frequency. (i) SNR and SNDR versus the input voltage amplitude. 


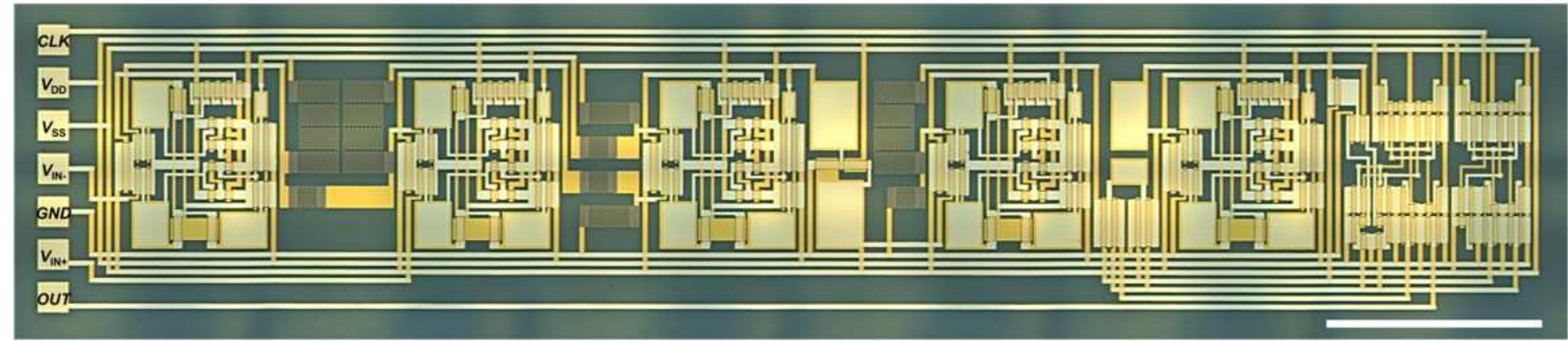

Instrumentation Amp.

BPF Non-inv. Amp.

$\Delta \Sigma \mathrm{A} / \mathrm{D}$ Converter

b
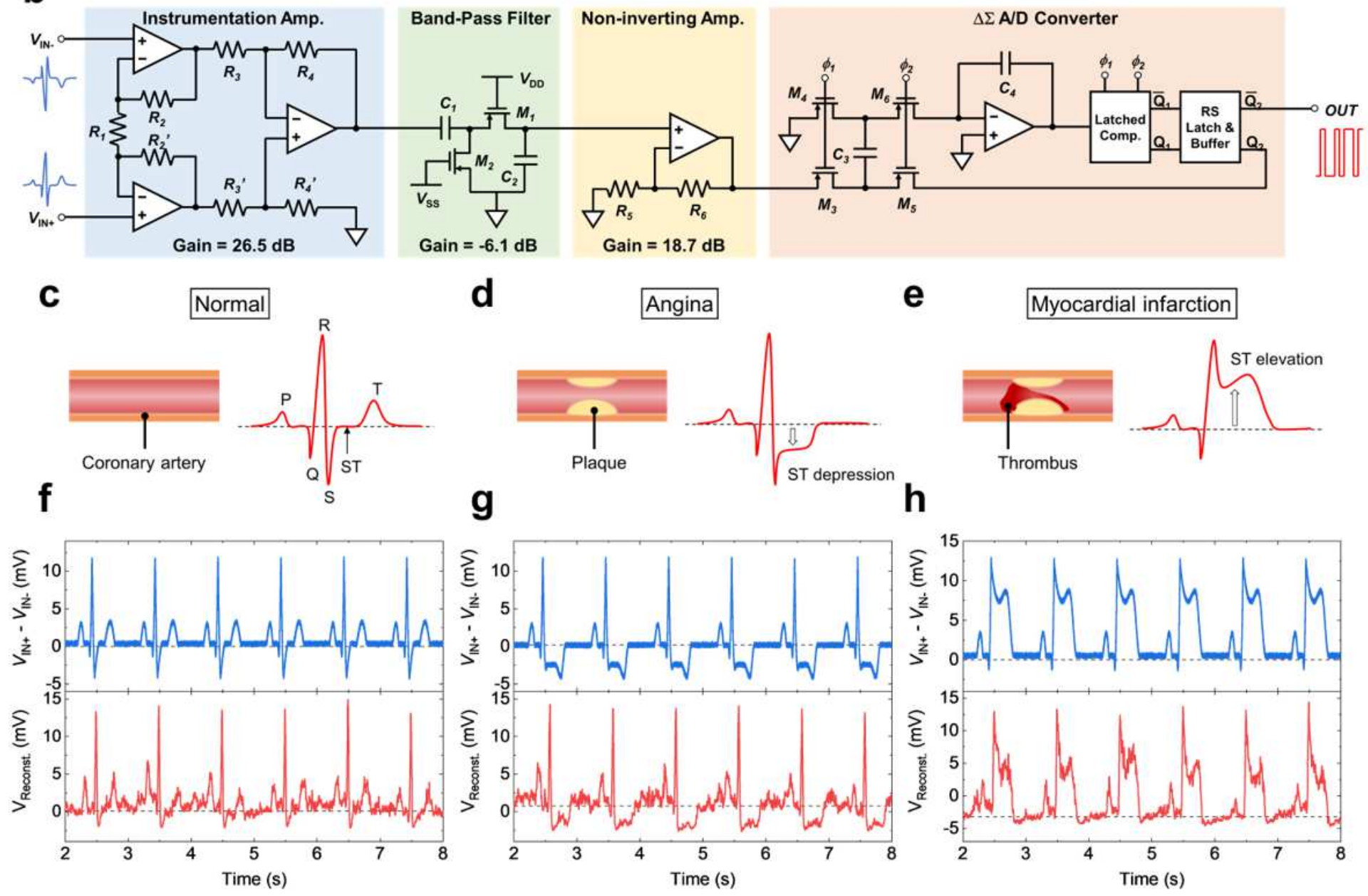

Figure 6

CNT-based fully flexible AFE. (a) Photograph (scale bar: $1 \mathrm{~mm}$ ) and (b) block diagram. Schematics of the coronary artery and ECG waveforms indicating (c) normal conditions, (b) an angina, and (c) a myocardial infarction (c). Input voltage waveforms to the AFM (upper) and reconstructed voltage waveforms from the digital output data (bottom) indicating (f) normal conditions, $(\mathrm{g})$ an angina, and (h) a myocardial infarction. 


\section{Supplementary Files}

This is a list of supplementary files associated with this preprint. Click to download.

- SupplementrayInformation200830submitted.docx 